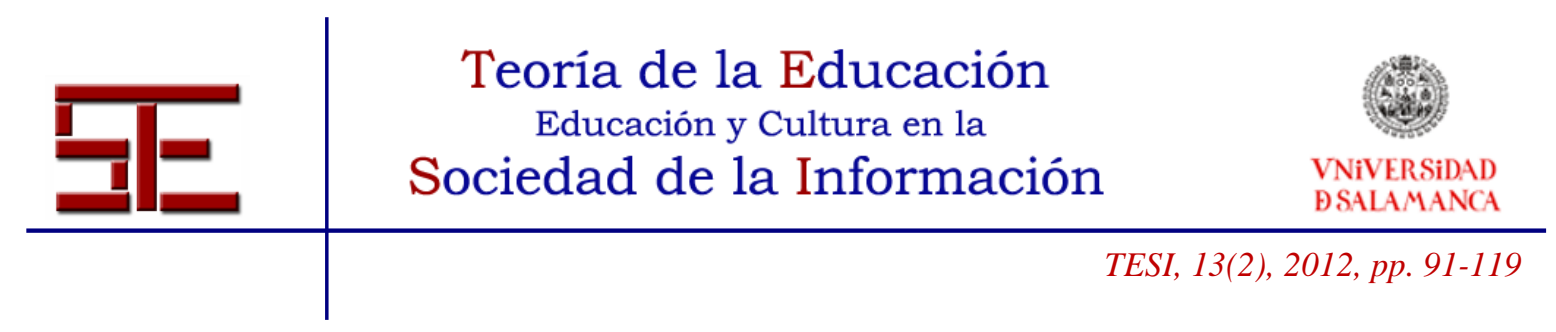

\title{
PROPUESTA COMUNITARIA CON ROBÓTICA EDUCATIVA: VALORACIÓN Y RESULTADOS DE APRENDIZAJE
}

Resumen: El proyecto busca diversificar la oferta educativa de niños, niñas y adolescentes que viven en condiciones de pobreza y riesgo social, mediante una propuesta didáctica con robótica educativa en la que las personas participantes diseñan, construyen y programan prototipos robóticos ambientados en situaciones reales. Las construcciones se hacen con bloques y operadores mecánicos (LEGO) y se programan con un lenguaje iconográfico (Robolab 2.9). La propuesta se enfoca en los procesos de diseño, construcción, programación y la divulgación de resultados y productos. La ejecución y evaluación de la propuesta y de los resultados de aprendizaje se realizó en tres centros comunitarios (Centros Interactivos), que son centros de aprendizaje no formales, ubicados en comunidades urbano marginales. Se observó que al finalizar los talleres los estudiantes elaboran proyectos grupales que representan sitios o eventos, utilizan mecanismos integrados por diferentes máquinas y operadores para la transmisión de movimiento, los programas para sus prototipos incluyen estructuras de multitareas y ciclos condicionados, estructuras de control de tiempo y uso de sensores. Sin embargo, se presentaron dificultades con el logro de los componentes sociales: trabajo en equipo, comunicación y socialización de los procesos de aprendizaje, que deberán ser abordados con mayor profundidad en una próxima fase.

Palabras clave: robótica educativa; habilidades tecnológicas; educación para población menor de edad.

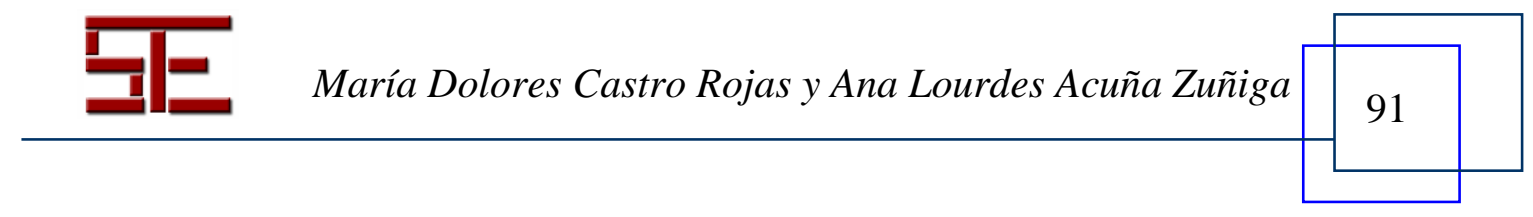




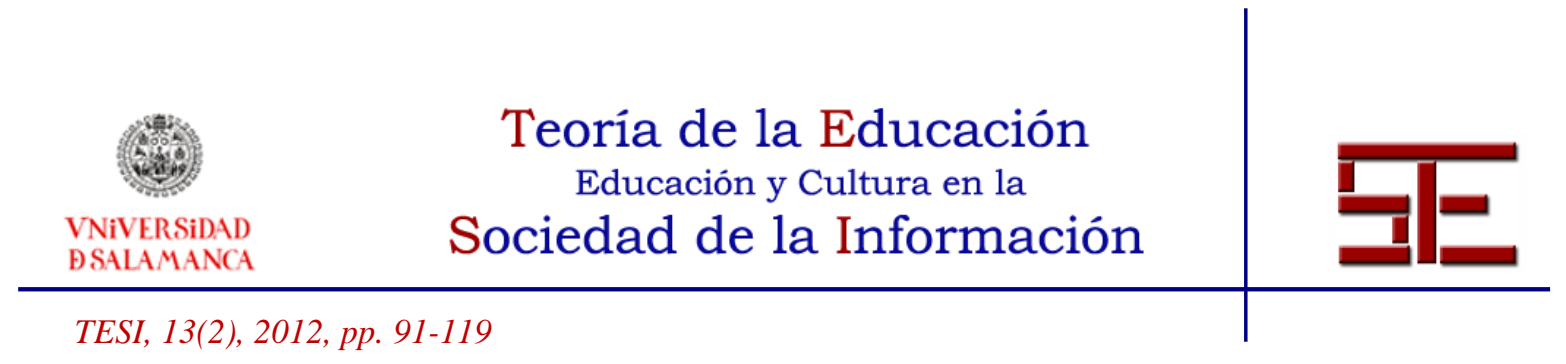

\title{
COMMUNITARIAN EDUCATIVE PROPOSAL WITH ROBOTICS: EVALUATION AND LEARNING PROCESS OUTCOMES
}

\begin{abstract}
The project seeks to expand young people's educative horizons that live in conditions of poverty and social risk, by means of an innovative didactic proposal integrating robotics. In this way the participants design, construct and program prototypes in real situations giving them new ways to experiment and share a significant experience. Their building representations are constructed with mechanical LEGO bricks and controlled by iconographic language (Robolab 2,9).
\end{abstract}

In fact, it was developed in three communitarian centers, which are no formal centers of schooling, located in urban marginal communities. As part of the evaluative process it was observed that students might be much likely to work in groups to represent different environments or conventional events, integrating different mechanical systems to drive their robotics prototypes. Thus, students may incorporate automatic responses, as well as programming structures such as multitask, conditional loops, time control and use of sensors.

For many young students of these communities there were over and over again constant social difficulties such as teamwork, lack of socialization skills and deficient formative experiences that we must pay attention to which ideas from our field do help these students build effective projects and significant robotics classroom.

Keywords: educative robotics; digital skills; technology for children.

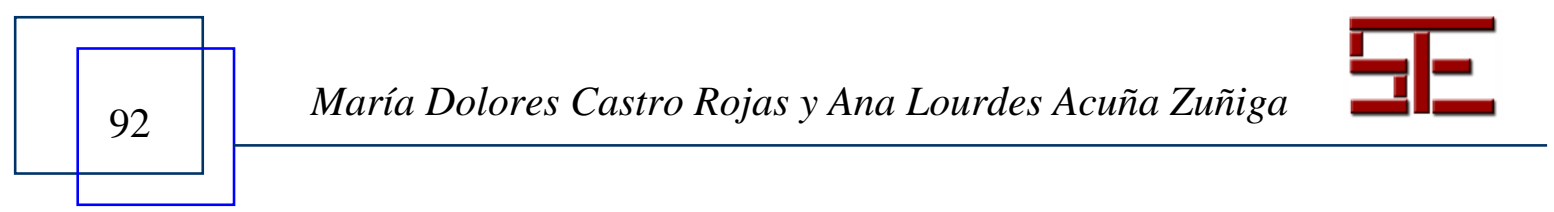




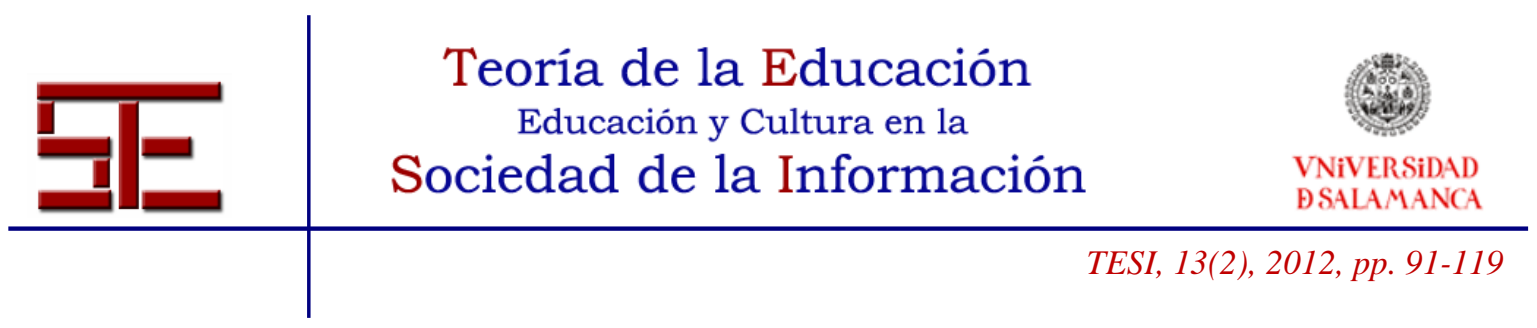

\section{PROPUESTA COMUNITARIA CON ROBÓTICA EDUCATIVA: VALORACIÓN Y RESULTADOS DE APRENDIZAJE}

Fecha de recepción: 09/05/2012; fecha de aceptación: 03/07/2012; fecha de publicación: 26/07/2012

\section{María Dolores Castro Rojas \\ mdolores.castro@fod.ac.cr \\ Fundación Omar Dengo \\ Ana Lourdes Acuña Zuñiga \\ mdolores.castro@fod.ac.cr}

Fundación Omar Dengo

\section{1.- INTRODUCCIÓN}

El proyecto es una iniciativa conjunta de la Fundación Omar Dengo Costa Rica y FundaVida, una ONG que brinda oportunidades a personas menores de edad que viven en zonas marginales de la provincia de San José. Busca diversificar y ampliar la oferta de aprendizaje de los Centros Interactivos de FundaVida, mediante la implementación de una propuesta didáctico-pedagógica de robótica educativa y la inclusión de otras opciones de aprendizaje como emprendimiento y herramientas de productividad, a las que puedan optar los niños, niñas y adolescentes en condiciones de pobreza y riesgo social, con el fin de mantenerlos en ambientes seguros que los alejen de las calles y los peligros del contexto en el que se desarrollan. Los niños, niñas y adolescentes asisten a los Centros Interactivos (CI) de manera voluntaria y en horario adicional a sus procesos de educación formal, los procesos de aprendizaje que allí se desarrollan resultan complementarios al sistema educativo formal, en el cual se fomenta que los estudiantes permanezcan.

La idea para hacer este proyecto fue premiada a finales del año 2010 con el HP EdTech Innovators Award, un programa de la empresa Hewlett-Packard, para la innovación social en educación alrededor del mundo, el cual consistió en la donación de $\$ 40.000$ usd en equipo tecnológico, con el cual se ha ejecutado la propuesta.

Durante el año 2011 se realizó la primera fase de implementación del proyecto de robótica educativa, que contempló en el diseño de la propuesta pedagógica y didáctica y de los materiales de aprendizaje, la capacitación de los docentes y la ejecución de los talleres en tres comunidades urbanas marginales de la capital.

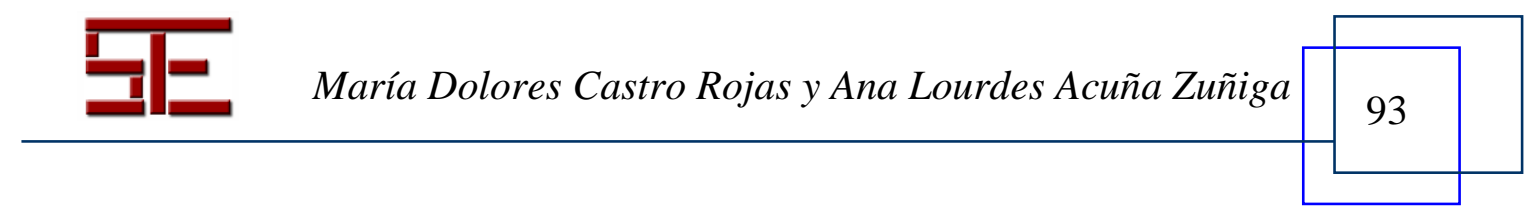




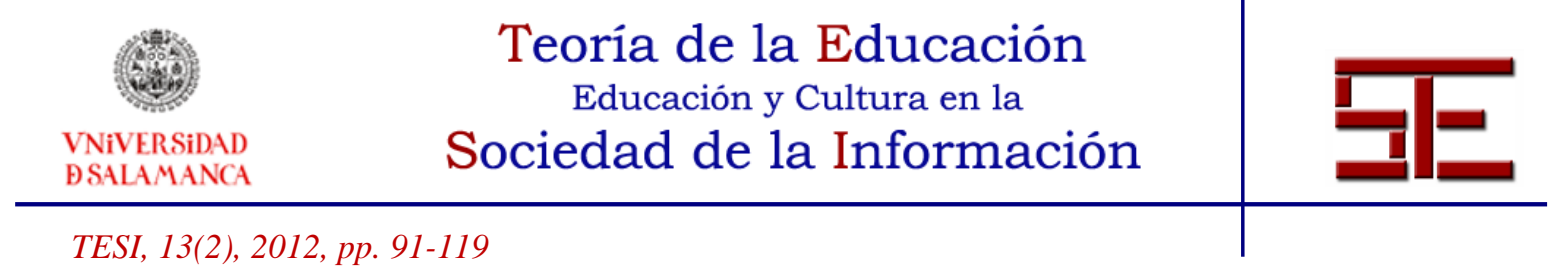

Los resultados obtenidos mediante el proceso de monitoreo y evaluación se enfocan en dos aspectos fundamentales: la puesta en práctica y adaptación de la propuesta de aprendizaje y los resultados de aprendizaje logrados por los y las participantes.

\section{2.- CONTEXTUALIZACIÓN DE LA PROPUESTA EDUCATIVA}

La robótica educativa, surge de las investigaciones y desarrollos emprendidos en los años 60 por Seymour Papert y otros investigadores del Laboratorio de Medios del Massachussets Institute of Tecnology (MIT) quienes crearon dispositivos tecnológicos que permitían a los niños construir edificios y máquinas, el trabajo se inició en colaboración con la compañía LEGO® para vincular los bloques de construcción con el lenguaje de programación Logo (Resnick, 2001) y en la década de los 80, esos juguetes ya se habían difundido por el mundo, incluyendo las escuelas, y junto a ellos las interrogantes sobre cómo obtener provecho en los procesos de aprendizaje de los estudiantes.

Así, en 1993, Seymour Papert, creador del construccionismo, disciplina que concede especial importancia al papel que pueden desempeñar las construcciones en el mundo como apoyo de las que se producen en la cabeza, propone la creación de una nueva materia: la "Cibernética", en la que se busca que el conocimiento se valore por la utilidad, por ser compatible con los demás y por adecuarse al estilo personal de cada uno (Papert, 1993). La “Cibernética para niños” de Papert, o la Robótica Educativa para nosotros, es una disciplina que se caracteriza por plantear:

Un marco para que los niños hagan inteligencia artificial, creen prototipos que superan lo humano porque incluyen animales y robots que van más allá de la realidad dejando espacio para la fantasía y (...) el uso de las nuevas tecnologías para hacer algo que nunca antes se había hecho (...) En el futuro los niños crecerán construyendo modelos cibernéticos con la misma facilidad que hoy se construyen coches, casas y trenes. Sólo entonces el pensamiento cibernético será parte de la cultura (Papert, 1993, 199-211).

Partiendo de las premisas propuestas por Papert y otros investigadores, el Área de Aprendizaje Lógico, Científico y Robótica de la Fundación Omar Dengo concibe la Robótica Educativa como un contexto de aprendizaje que se apoya en las tecnologías digitales y en los procesos de mediación pedagógica para que los estudiantes creen prototipos o simulaciones robóticas que surgen a partir del ingenio, la creatividad y puesta en práctica de los conceptos aprendidos. Los participantes diseñan, construyen y programan creaciones propias primero mentales y luego físicas-externas, construidas con diferentes materiales y controladas por un computador a través de un lenguaje de programación.

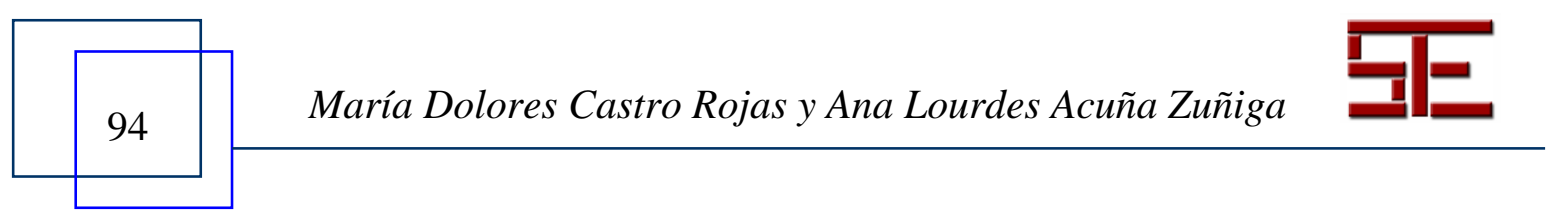




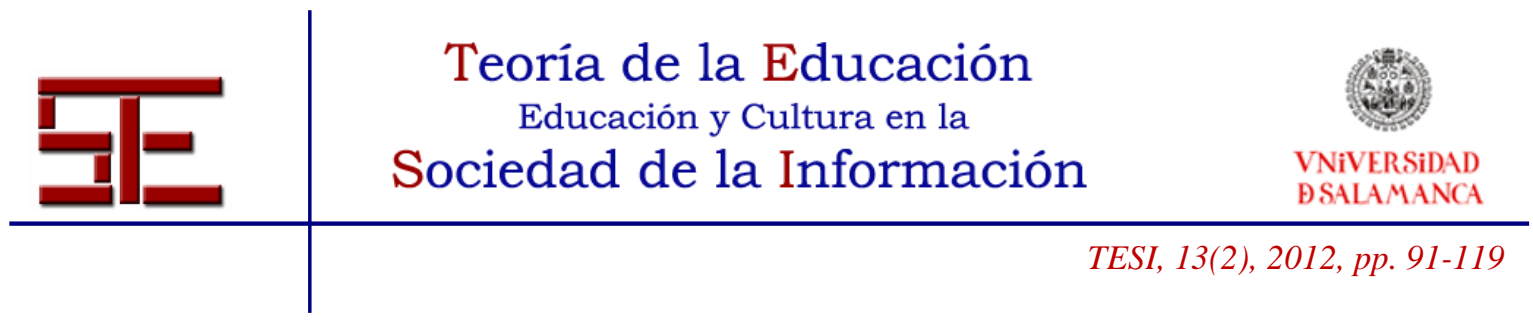

Esas creaciones pueden originarse a partir del estudio de realidades, por ejemplo, la apariencia o las formas de movimiento e interacción con el ambiente de mecanismos o máquinas, los componentes de un proceso de producción, un sitio específico o un ambiente particular con las interacciones entre los distintos elementos que lo integran. Otros productos pueden ser prototipos que corresponden a réplicas de diseños originales inspirados a partir del estudio de una situación problema particular o de la imaginación y creatividad personal o grupal de los integrantes en ese ambiente de aprendizaje (Acuña, 2006).

\section{3.- PROPUESTA DIDÁCTICO-PEDAGÓGICA PARA ROBÓTICA EDUCATIVA EN CENTROS COMUNITARIOS}

Una propuesta didáctico-pedagógica para robótica educativa a impartir en modalidad taller presencial que privilegia un proceso de aprendizaje centrado en el estudiante que se desenvuelve, aprende, convive y se desarrolla integralmente en su comunidad. Se considera al estudiante como persona capaz de trascender sus propias limitaciones, con identidad propia y con grandes capacidades creativas y de resolución de problemas y situaciones. Asimismo, el educador se concibe como un mediador del proceso de aprendizaje, dispuesto a aprender, a estudiar y a analizar su práctica educativa de forma permanente desde un marco analítico y valorativo en busca del éxito conjunto con sus estudiantes.

Se proyecta que quienes participen como aprendices de esta propuesta educativa comprendan los principios fundamentales de la robótica y del diseño de proyectos e identifiquen y pongan en práctica estrategias de construcción, programación y control de prototipos robóticos de invención propia. Además se aspira a que como grupo los estudiantes mejoren sus procesos de comprensión y resolución de problemas y comuniquen por diferentes medios sus rutas de aprendizaje y de producción. Bajo estas premisas se identificaron cuatro procesos de aprendizaje sobre los cuales se enfocaría la propuesta educativa: construcción, programación, diseño y publicación. Para cada uno de ellos se anticipó un conjunto de habilidades que se esperaba cultivar y observar en la población meta durante la ejecución de la propuesta y fueron los derroteros de los procesos de capacitación, de evaluación y monitoreo ejecutados. En cada uno de estos procesos se espera de los estudiantes lo siguiente:

Habilidades de construcción: Identifican los principios constructivos y mecánicos que les permiten crear y poner a funcionar prototipos robóticos de invención propia.

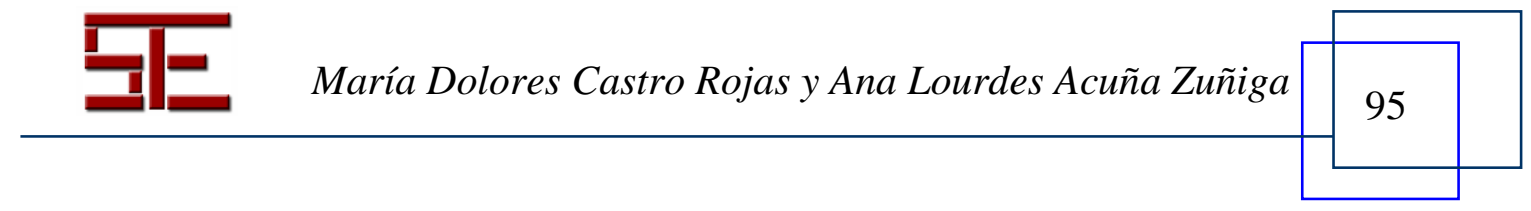




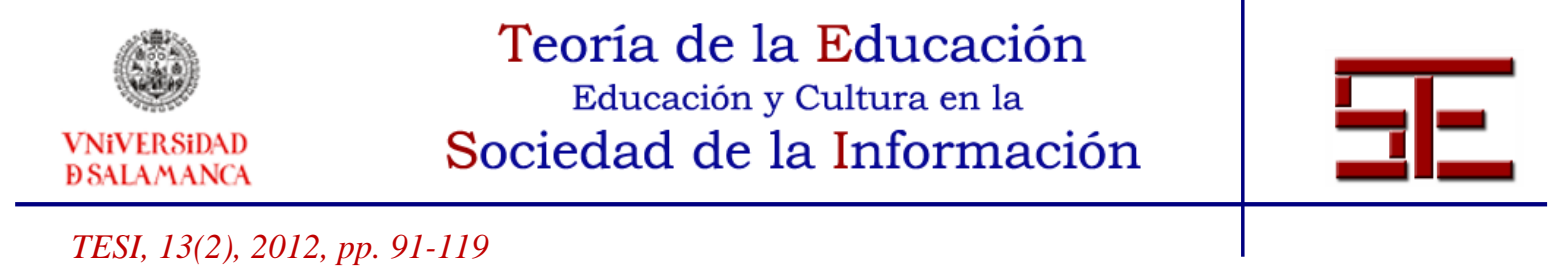

Habilidades de programación: Programan sistemas de control para sus robots que permiten un óptimo funcionamiento de acuerdo a su estructura y función.

Habilidades creativas: Concretan proyectos que simulan sitios y eventos que integran prototipos robóticos en la representación de situaciones o problemas de la vida real.

Habilidades sociales: Evidencian estrategias de resolución de problemas tanto a nivel tecnológico como de convivencia y aprendizaje con sus pares; evidencian trabajo en equipo en sus procesos de aprendizaje y en la concreción de proyectos; comparten sus procesos de pensamiento en forma verbal o gráfica, y divulgan sus productos y procesos de aprendizaje en forma presencial y digital.

Por otra parte, esta propuesta educativa privilegia las experiencias de aprendizaje que promueven la colaboración entre los pares como estrategias que coadyuvan a la construcción del aprendizaje significativo, el fomento del trabajo en equipo y el intercambio de ideas. Así como, la realización de proyectos que involucran a todos los miembros que interactúan en el ambiente de aprendizaje se constituye en un recurso de representación, y evidencia la concreción de aprendizajes y habilidades particulares, de igual manera el trabajo en parejas se constituyó en un medio que fomenta el intercambio de ideas y de formas de pensamiento. Además promueve competencias comunicativas a través del texto, el dibujo y la expresión oral y escrita en medios tradicionales y digitales.

La dinámica de las sesiones incluye un componente teórico o conceptual y su respectiva aplicación práctica con pequeños ejercicios o retos durante las sesiones. También se contempla la realización de proyectos que sirven como fuentes de verificación del aprendizaje de los estudiantes: un pequeño proyecto intermedio realizado en parejas y un proyecto final grupal más complejo realizado por todos los integrantes del grupo, ambas producciones se exponen a un público invitado que pueden ser familiares o miembros de la comunidad en la que residen los estudiantes.

Asimismo, se consideraron las condiciones de espacio, tiempo, cantidad de beneficiados y recursos tecnológicos disponibles para la ejecución efectiva de la propuesta educativa y el logro de las habilidades propuestas. Por esas razones se estableció un marco de ejecución inicial de 10 sesiones (una por semana) de 3 horas cada una, con grupos de 16 estudiantes, utilizando equipos de construcción (LEGO) por bloques con operadores

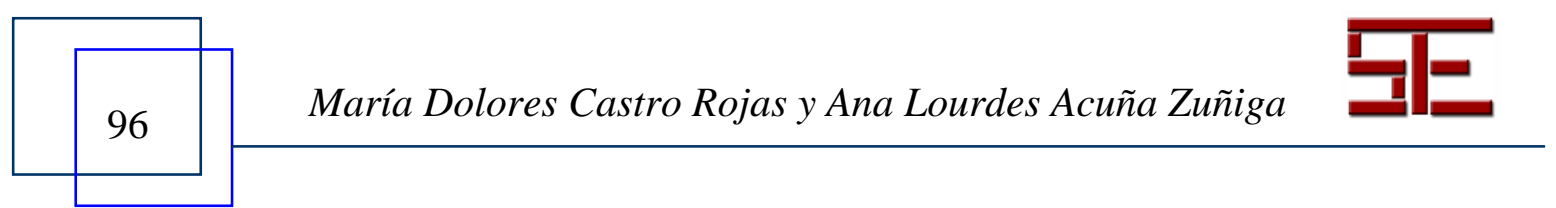




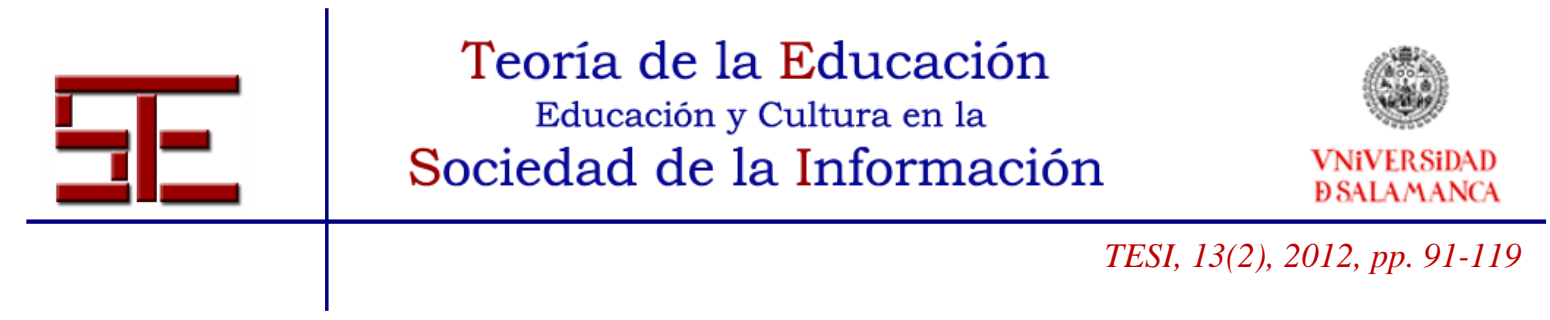

mecánicos que faciliten la creación de mecanismos y de máquinas simples y compuestas. Asimismo, se seleccionó un lenguaje de programación iconográfico (Robolab 2.9) que facilitara los procesos lógicos, de pensamiento e interactividad con el usuario por medio de sensores. Intentando disminuir las dificultades sintácticas que algunos lenguajes de programación para robótica tienen y que entorpecen los procesos de programación y de pensamiento en los estudiantes de edad escolar.

El siguiente gráfico muestra la integración de los procesos de diseño, construcción, programación y divulgación, respecto al tiempo de ejecución de la propuesta. Cabe destacar que los procesos de divulgación ocurren en forma presencial al completar la primera mitad del taller y al finalizar el taller, inicialmente esto ocurría en la $\mathrm{V}$ y $\mathrm{X}$ semana. Además se espera una publicación web al final de cada taller.

Gráfico 1. Procesos de propuesta educativa y distribución en las sesiones de trabajo propuestas

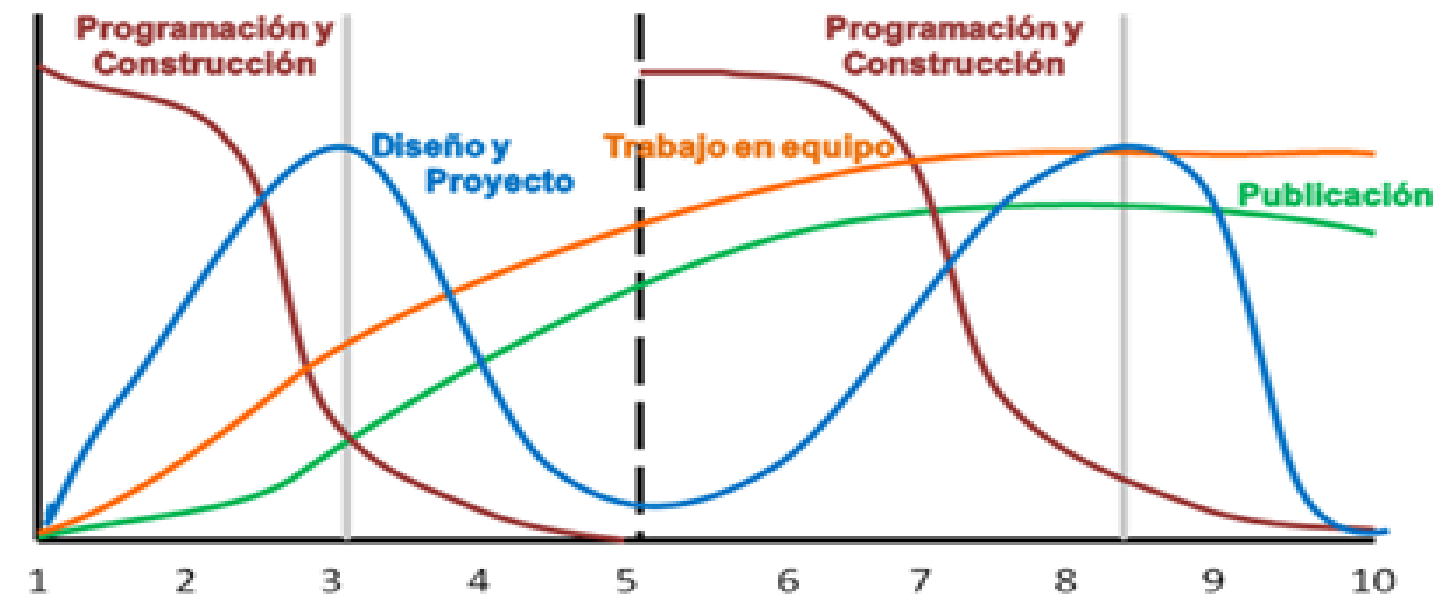

Nota: En el cuerpo del gráfico se observan los procesos de aprendizaje que se desarrollan y en la base la distribución temporal para las 10 sesiones de trabajo propuestas originalmente.

Esta propuesta inicial fue ejecutada en tres ocasiones durante el año 2011 y validada a partir de la realimentación del equipo docente de FundaVida la opinión de los estudiantes y de los resultados de aprendizaje obtenidos en la población meta. Aunque inicialmente se planteó un proceso de 10 sesiones con una duración de 3 horas cada, con el fin de abordar con más detalle y profundidad algunos contenidos se amplió el número

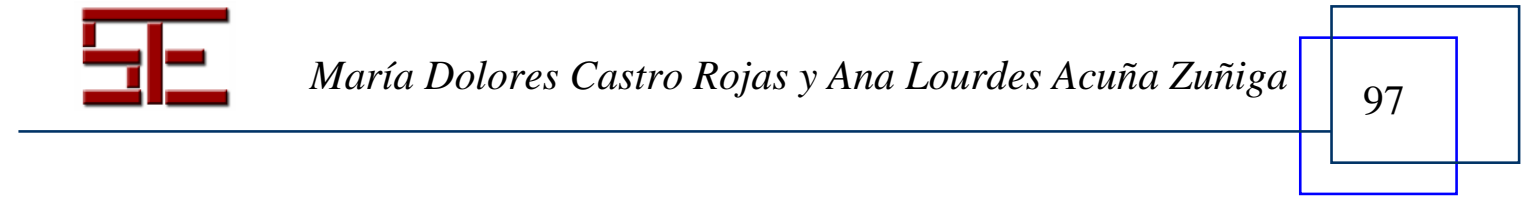




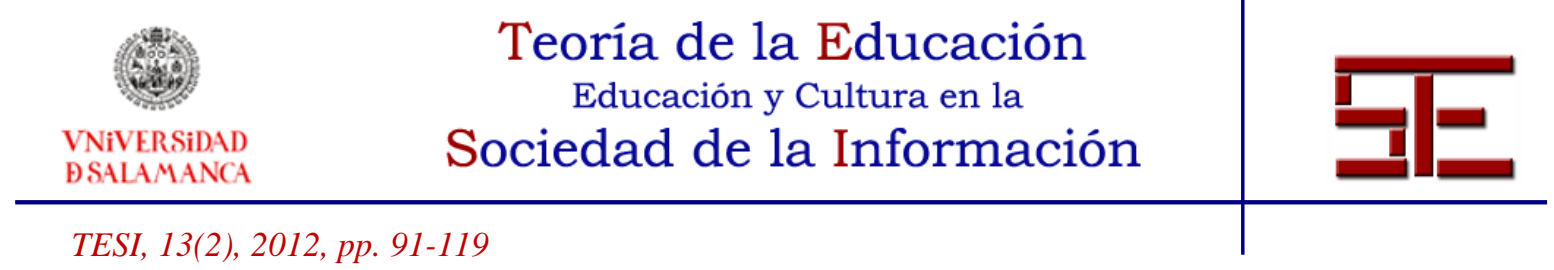

a 12 sesiones se excluyeron algunos temas de la propuesta inicial dejándolos para una próxima propuesta y se ratificó un tiempo efectivo de trabajo en robótica de 2 horas por sesión.

Una vez establecida la propuesta educativa, se definieron dos cortes evaluativos en los cuales los estudiantes exponen su trabajo en público. Para la primera exposición se espera que los estudiantes, en parejas de trabajo, rediseñen y contextualicen una construcción previa que hayan realizado durante las actividades de práctica e integren los prototipos en un proyecto que representa un sitio sobre alguna temática específica. En este punto no se espera un proyecto muy complejo sino más bien una representación que muestre el avance del proceso de aprendizaje y puesta en práctica de algunos conceptos básicos. Para la exposición final el producto esperado es un proyecto planeado y diseñado de manera grupal, en el que los y las participantes eligen de manera consensuada una temática para representar un sitio o un evento, construyen y programan proyectos complejos en los que cada subgrupo o pareja participa de manera activa creando prototipos que cumplen funciones específicas dentro de la situación recreada, por lo que el proyecto debe ser coordinado entre todos los estudiantes que conforman el grupo en general.

Previo al inicio de la implementación de la propuesta se realizaron sesiones de trabajo conjunto con las docentes de FundaVida con la finalidad de valorar la propuesta educativa diseñada e identificar los temas en los que requerían mayor capacitación. Posteriormente, los educadores participaron en un taller de 40 horas que les involucró en el estudio y comprensión de los principios básicos de la robótica educativa y luego se planearon sesiones de estudio particulares para abordar temas como estrategias de mediación para el diseño de proyectos, máquinas compuestas, grados de libertad y movimientos excéntricos, metodológicas y didácticas para la enseñanza de la robótica. Conforme se fueron implementando las diferentes sesiones los educadores identificaron materiales didácticos que resultaban más útiles y de mejor comprensión para los estudiantes. También se hicieron cambios en el orden y abordaje de algunos temas y del tiempo que se les dedicaba para su estudio.

\section{4.- RESULTADOS}

La evaluación y adaptación de la propuesta educativa y de los resultados de aprendizaje obtenidos por los estudiantes se realizó con una rúbrica que para la primera ejecución de la propuesta incluyó el eje de construcción, con las dimensiones: 1) Diseño, 2) Conocimiento y utilización de piezas de construcción, 3) Construcción de mecanismos, y 4) Resolución de problemas de construcción; y el eje de Programación con las

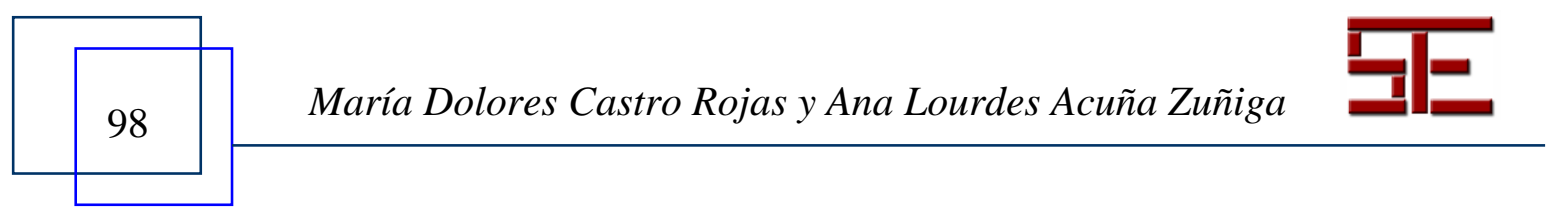




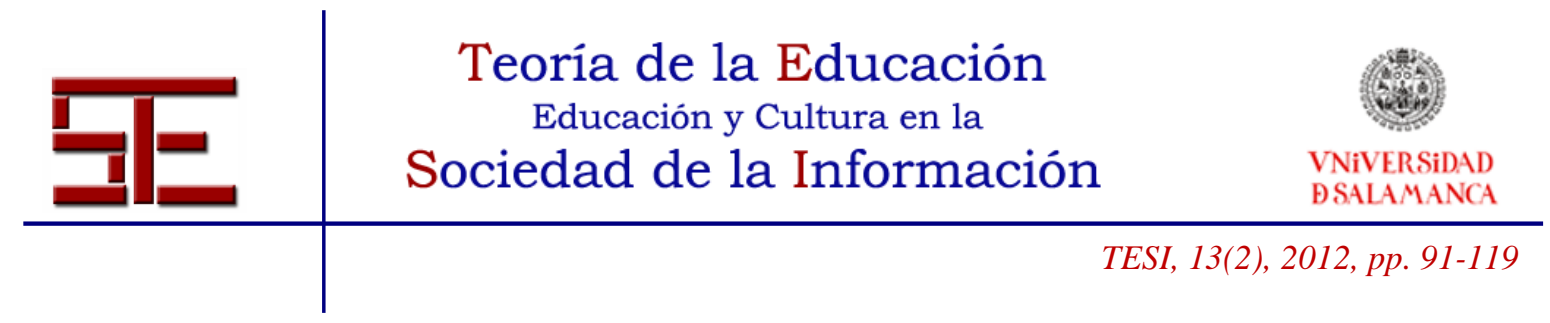

dimensiones: 1) Control de actuadores, 2) Uso de estructuras de control y 3) Resolución de problemas de programación.

La rúbrica se construyó de manera que se asignara un puntaje máximo a cada dimensión de los ejes evaluados: el valor máximo 4 refleja el dominio de la dimensión evaluada y el valor mínimo 1 refleja poco dominio. Cada valor fue operacionalizado en criterios específicos.

Sin embargo, después de la primera sesión de evaluación se concluyó que debían ampliarse los ejes de evaluación de la rúbrica de manera que coincidieran de forma más precisa con la propuesta de aprendizaje y las habilidades por alcanzar, por lo que para la segunda y tercera aplicación la rúbrica incluyó los siguientes ejes y dimensiones:

Construcción: 1) Conocimiento y utilización de las piezas de construcción, 2) Construcción de mecanismos, 3) Acople de sistemas de engranajes, 4) Resolución de problemas de construcción y 5) Uso del tiempo.

Programación: 1) Control de actuadores, 2) Resolución de problemas de programación, 3) Uso de estructuras de control de tiempo, 4) Uso de estructuras de programación (estructuras simples), 5) Ciclos condicionados, estructuras iterales y condicionales, 6) Programación de tareas (multitareas), 7) Uso de diversidad de comandos y 8) Efectividad en la programación.

Trabajo en equipo: 1) Colaboración y apoyo, 2) Contribuciones de los integrantes, 3) Concentración en la tarea, 4) Esfuerzo y 5) Comunicación.

Proyecto grupal: 1) Diseño previo, 2) Temática y 3) Diversidad de prototipos.

Exposición del trabajo: 1) Claridad, orden y 2) Organización de la información.

Se mantuvo el rango de evaluación entre 4 y 1 . Para ilustrar la rúbrica de evaluación en la Figura 1 se muestra la evaluación del eje construcción y la dimensión "Construcción de mecanismos".

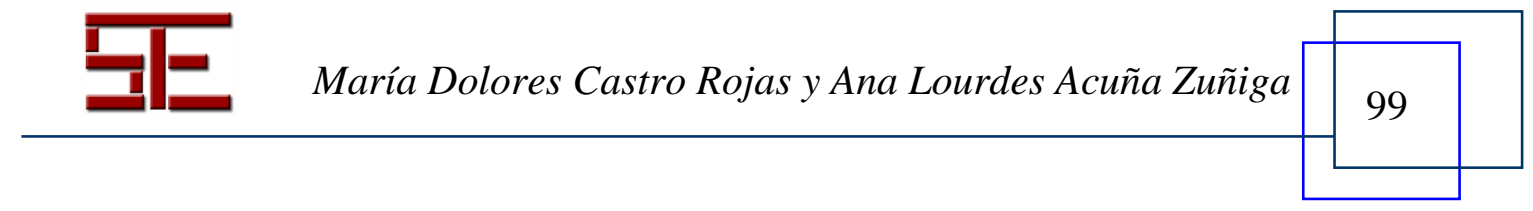




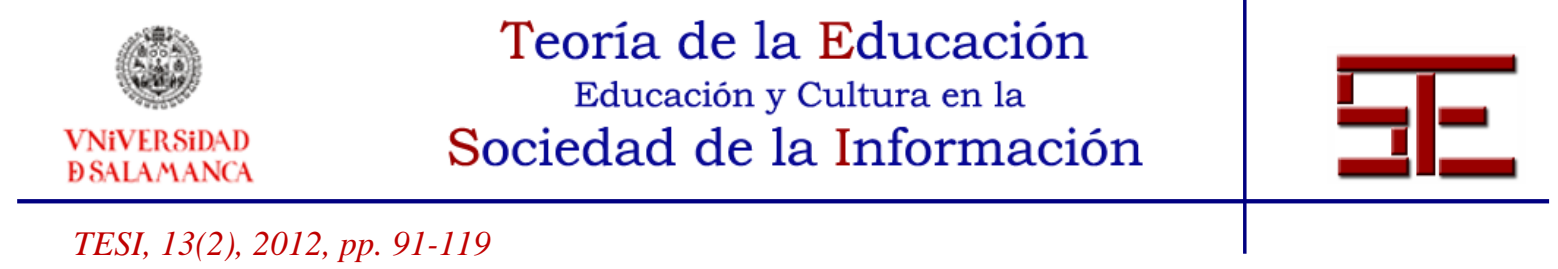

Figura 1. Fragmento de rúbrica para el eje de construcción y la dimensión construcción de mecanismos

\begin{tabular}{|c|c|c|c|c|}
\hline & 4 & 3 & 2 & 1 \\
\hline $\begin{array}{l}\text { Construcción } \\
\text { de mecanismos }\end{array}$ & $\begin{array}{lr}\text { El proyecto } & \text { cuenta } \\
\text { con dos o más } \\
\text { mecanismos } & \text { que } \\
\text { integran diferentes } \\
\text { operadores, y los } \\
\text { utiliza para la } \\
\text { transmisión } & \text { de } \\
\text { movimiento } & \text { en } \\
\text { diferentes planos. }\end{array}$ & $\begin{array}{l}\text { El proyecto cuenta } \\
\text { con un mecanismo } \\
\text { que } \\
\text { diferentes } \\
\text { operadores. }\end{array}$ & $\begin{array}{l}\text { El proyecto cuenta } \\
\text { con un mecanismo, } \\
\text { pero no cumple su } \\
\text { función en la } \\
\text { transmisión } \\
\text { movimiento. }\end{array}$ & $\begin{array}{l}\text { El proyecto no posee al } \\
\text { menos un mecanismo } \\
\text { que funcione } \\
\text { correctamente. }\end{array}$ \\
\hline
\end{tabular}

En cada sesión de evaluación la valoración fue realizada por dos personas, una que se desempeñó como docente durante las tres ejecuciones de la propuesta y la otra fue un funcionario o funcionaria del Área de aprendizaje lógico, científico y robótica de la Fundación Omar Dengo. Esto con el fin de poder contrastar la información de ambas fuentes. Los resultados finales se procesaron con la información de ambos evaluadores utilizando el paquete estadístico SPSS 19. A continuación se presentan las medias alcanzadas por los estudiantes en cada dimensión evaluada.

\subsection{Participantes}

Durante el año 2011 participaron un total de 79 niños, niñas y adolescentes, 47 varones y 32 mujeres, con una media de edad de $\mathrm{ME}=11.54$ años, $\mathrm{DE}=1.42$, todos los participantes se encontraban cursando estudios formales al momento de asistir a los talleres de robótica educativa. El horario de los talleres de aprendizaje en las sedes de FundaVida se ubica fuera de la jornada escolar y los estudiantes asisten de manera voluntaria. El detalle de los y las participantes se muestra en la Tabla 1.

Tabla 1. Cantidad de participantes por Centro Interactivo durante el 2011

\begin{tabular}{cccc}
\hline Centro Interactivo & Total de participantes & Hombres & Mujeres \\
\hline 25 de julio, Hatillo & 27 & 19 & 8 \\
Concepción Abajo de Alajuelita & 25 & 13 & 12 \\
Linda Vista, Patarrá & 27 & 15 & 12 \\
TOTALES & 79 & 47 & 32 \\
\hline
\end{tabular}

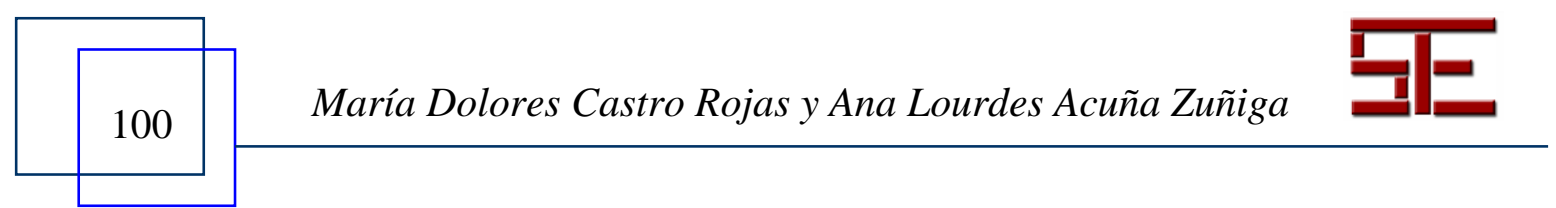




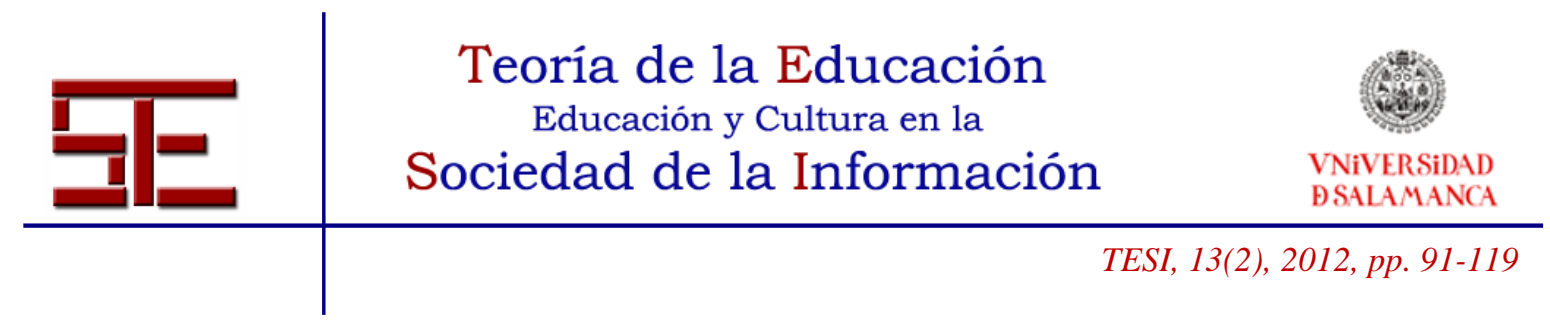

Cabe aclarar que además de las evaluaciones de proyectos realizadas a mitad de cada taller y en las sesiones finales se realizaron observaciones durante las sesiones ordinarias de trabajo.

\section{2 . Resultados obtenidos en las primeras exposiciones}

En las tres ejecuciones de la propuesta para la primera exposición los estudiantes se inclinaron por representar desfiles que surgen a partir de la contextualización de una situación a representar y de la incorporación de los móviles que tienen construidos hasta la fecha, pero acondicionados según la situación y las consignas sugeridas en la propuesta educativa.

Los resultados obtenidos en los proyectos y al momento de la exposición se presentan organizados de acuerdo a los procesos de aprendizaje.

\subsubsection{Construcción}

En general al finalizar la primera mitad del taller los estudiantes son capaces de construir prototipos robóticos simples, en la mayoría de los casos carros, utilizando piezas de construcción genéricas, además construyen mecanismos simples para dar movimiento a sus prototipos y aplican conceptos muy básicos de reconocimiento de sistemas simples de fuerza y velocidad para lograr los efectos deseados en sus construcciones.

En la Tabla 2 se muestran los resultados para el eje de construcción. Se observa que a través de las distintas aplicaciones las medias en el ámbito de construcción fueron mejorando, esto se relaciona con los cambios en la propuesta educativa, la inclusión de mayor cantidad de lecciones y la adquisición de mayor dominio en el campo por parte del equipo docente.

Tabla 2. Resultados para la construcción en la primera exposición

\begin{tabular}{|c|c|c|c|c|c|c|c|}
\hline \multirow{4}{*}{ Aspecto } & \multicolumn{7}{|c|}{ Centros Interactivos } \\
\hline & \multicolumn{2}{|c|}{$\begin{array}{l}25 \text { de Julio, } \\
\text { Hatillo }\end{array}$} & \multicolumn{2}{|c|}{$\begin{array}{l}\text { Concepción de } \\
\text { Alajuelita }\end{array}$} & \multicolumn{3}{|c|}{$\begin{array}{c}\text { Linda Vista } \\
\text { Patarrá }\end{array}$} \\
\hline & \multirow{2}{*}{$\begin{array}{c}\text { M } \\
4 \text { Sesión }\end{array}$} & \multirow{2}{*}{$\begin{array}{c}\text { DE } \\
4 \\
\text { Sesión }\end{array}$} & \multirow{2}{*}{$\begin{array}{c}\text { M } \\
5 \\
\text { Sesión }\end{array}$} & \multirow{2}{*}{$\begin{array}{c}\text { DE } \\
5 \\
\text { Sesión }\end{array}$} & \multirow{2}{*}{\multicolumn{2}{|c|}{$\begin{array}{c}\text { M } \\
6 \\
\text { Sesión }\end{array}$}} & \multirow{2}{*}{$\begin{array}{c}\text { DE } \\
6 \\
\text { Sesión }\end{array}$} \\
\hline & & & & & & & \\
\hline \multirow[t]{2}{*}{ Conocimiento y utilización de } & 2.75 & .88 & 3.20 & .83 & \multicolumn{2}{|c|}{3.40} & .69 \\
\hline & es Castro $\mathrm{I}$ & ojas y $A n$ & Lourdes & cuña Zu & & 10 & \\
\hline
\end{tabular}




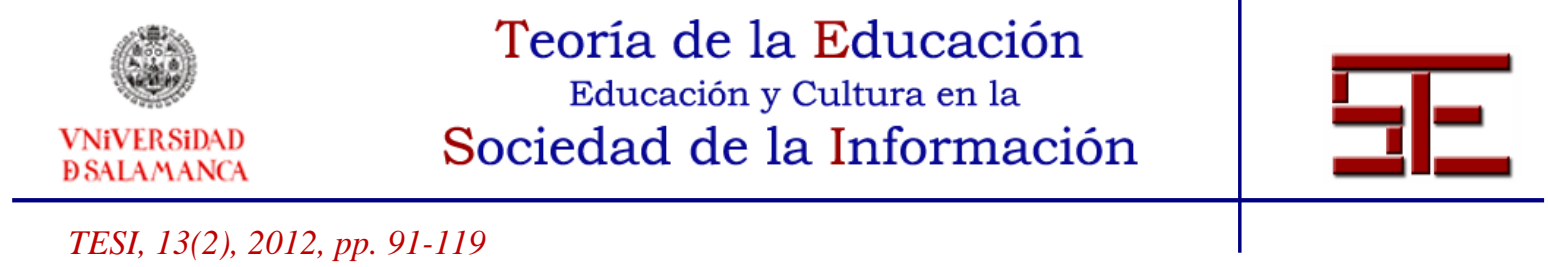

piezas de construcción

Utilización de mecanismos

2.50

.53

3.40

.89

3.80

.42

Utilización de sistemas de engranajes

Resolución de problemas de construcción

.83

3.60

En la tabla se aprecia que las medias en la segunda y tercera aplicación alcanzan valores cercanos al valor 4, que representa el desempeño ideal esperado, lo que indica que el desempeño de los estudiantes es satisfactorio y muy cercano a lo que se espera de ellos en un escenario ideal. Es decir, de manera global sus prototipos cumplen con las características y requerimientos solicitados.

Sin embargo, se observa que las medias más bajas refieren a la construcción de mecanismos, el acople de sistemas de engranajes y la resolución de problemas. Si bien es cierto que los estudiantes logran construir mecanismos simples para que sus prototipos cumplan con las funciones básicas que ellos desean, no logran desarrollar sistemas mecánicos compuestos que respondan a cabalidad con la tarea esperada, al interrogarles sobre esta limitación se pudo determinar que en este momento de los talleres todavía no comprenden los conceptos como fuerza, velocidad y sus efectos en la transmisión de movimiento, tampoco identifican los principios de acople que convierten un mecanismo simple en uno compuesto y que subyacen a los principios de construcción estudiados.

Por otra parte, dentro de la propuesta no se desarrolló de manera formal un enfoque de resolución de problemas ni de planificación o diseño previo que permita a los estudiantes desarrollar prever los problemas que se puedan presentar y generar las estrategias adecuadas para resolverlos.

Uno de los aspectos que mostró mejores medias conforme avanzaron las ejecuciones de la propuesta fue el conocimiento y utilización de piezas de construcción, lo que se tradujo en la construcción de prototipos más estables y robustos cada vez.

Dado que en la primera ejecución del taller no se utilizó la misma rúbrica de evaluación, no fue posible comparar las medias obtenidas en los talleres. No obstante, se realizó una prueba $t$ para muestras independientes, para los resultados de la segunda y la tercera

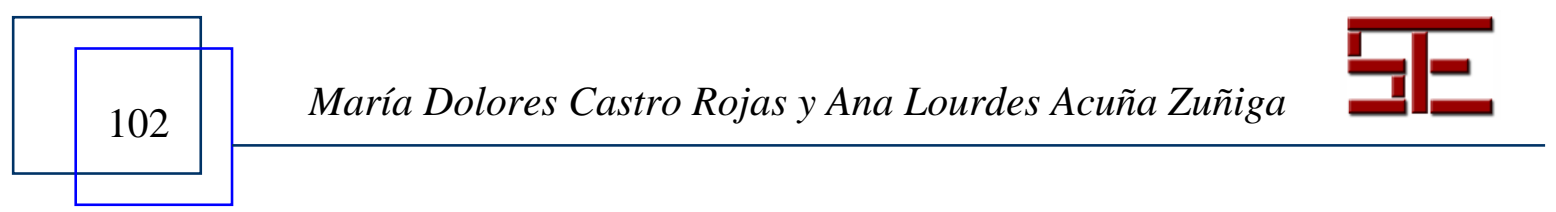




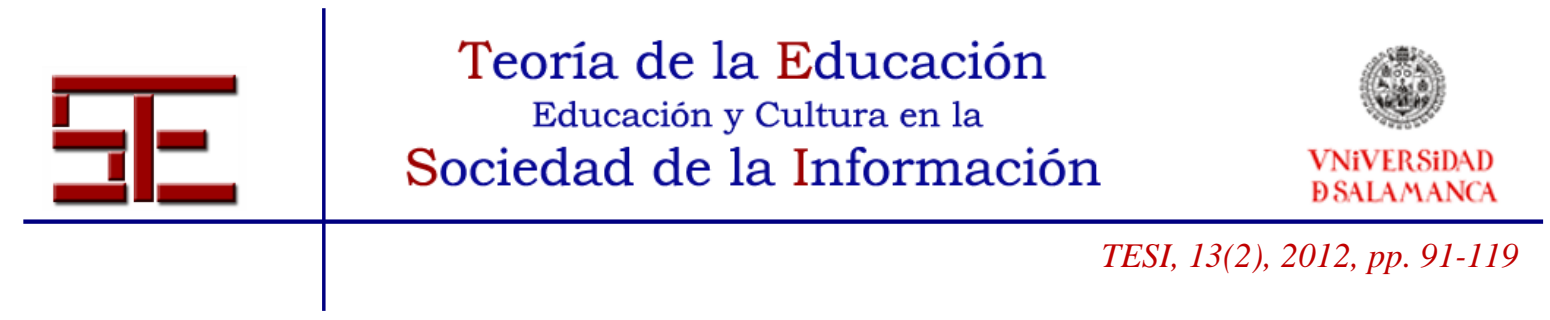

aplicación y no se evidenciaron diferencias estadísticamente significativas entre las medias. Es decir, el nivel de logro de los estudiantes se mantuvo constante a través de las distintas ejecuciones de la propuesta y en términos generales ese desempeño estuvo cercano a lo esperado en el área de construcción, evidenciando dificultades en la resolución de problemas.

\subsubsection{Programación}

Al finalizar la primera parte del taller los estudiantes logran programar estructuras simples para controlar actuadores (motores, lámparas, sirenas, servomotores) y asignar tiempos a las tareas programadas. Además, utilizan diversos comandos de programación y en gran medida logran los comportamientos deseados en sus prototipos. En las aplicaciones 2 y 3 las medias se ubican por encima del valor 3 y cercanas al valor 4 que es el máximo desempeño esperado en los estudiantes. El detalle de los resultados se muestra en la Tabla 3.

Tabla 3. Resultados para programación en la primera exposición

\begin{tabular}{|c|c|c|c|c|c|c|}
\hline \multirow{5}{*}{ Aspecto } & \multicolumn{6}{|c|}{ Centros Interactivos } \\
\hline & \multicolumn{2}{|c|}{$\begin{array}{l}25 \text { de Julio, } \\
\text { Hatillo }\end{array}$} & \multicolumn{2}{|c|}{$\begin{array}{c}\text { Concepción de } \\
\text { Alajuelita }\end{array}$} & \multicolumn{2}{|c|}{$\begin{array}{l}\text { Linda Vista } \\
\text { Patarrá }\end{array}$} \\
\hline & $\mathrm{M}$ & $\mathrm{DE}$ & $\mathrm{M}$ & $\mathrm{DE}$ & $\mathrm{M}$ & $\mathrm{DE}$ \\
\hline & 4 Sesión & 4 & 5 & 5 & 6 & 6 \\
\hline & & Sesión & Sesión & Sesión & Sesión & Sesión \\
\hline Programación de actuadores & 2.88 & 0.8 & 3.00 & .00 & 3.90 & .31 \\
\hline $\begin{array}{c}\text { Resolución de problemas de } \\
\text { programación }\end{array}$ & 1.25 & 0.7 & 3.20 & .44 & 3.60 & .69 \\
\hline $\begin{array}{l}\text { Programación de estructuras de } \\
\text { control de tiempo }\end{array}$ & 2.75 & 0.9 & 3.00 & .00 & 3.50 & .70 \\
\hline $\begin{array}{l}\text { Uso de estructuras de } \\
\text { programación }\end{array}$ & & & 2.00 & 1.00 & .00 & .00 \\
\hline Utilización de tareas & & & 2.20 & 1.09 & .00 & .00 \\
\hline Diversidad de comandos & & & 3.00 & .00 & 3.60 & .51 \\
\hline Efectividad & & & 2.75 & .50 & 3.70 & .48 \\
\hline
\end{tabular}

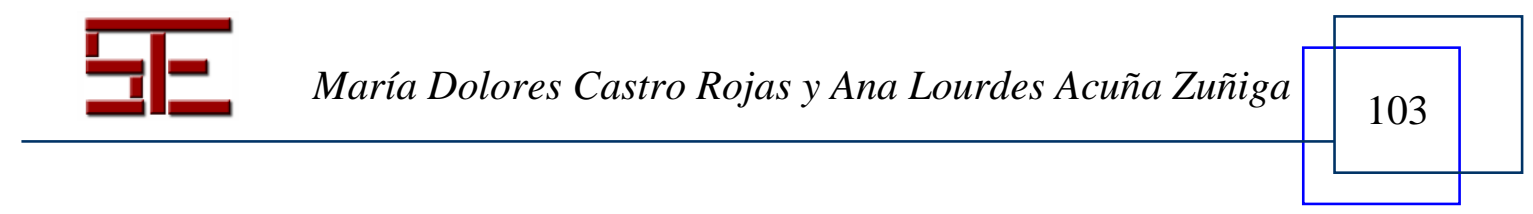




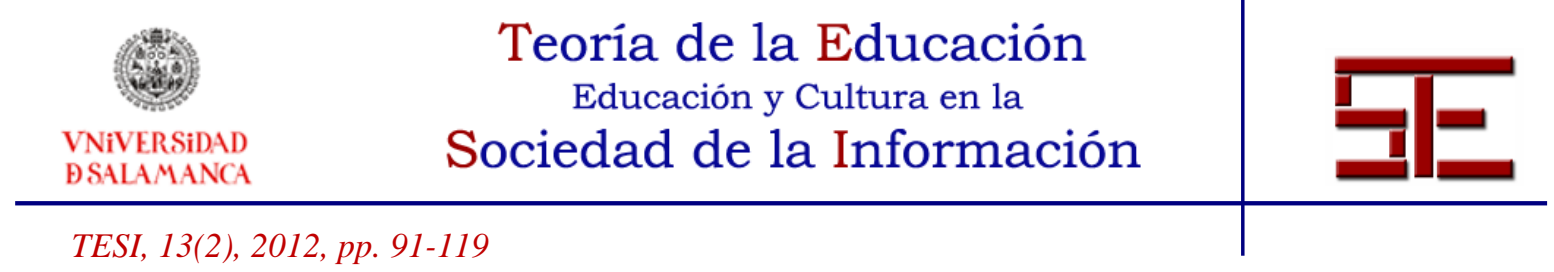

En términos generales la programación muestra medias más bajas que el componente de construcción, pues la programación o adquirir habilidades para programar ocurre de manera menos natural que la construcción y requiere más procesos de intervención y de resolución simultánea de problemas. Por sí misma la construcción tiene un componente lúdico y de entretenimiento para los y las estudiantes, quienes manifiestan que prefieren construir estructuras que programarlas, pues resulta más entretenido construir lo que deseen y cambiar sus construcciones de manera espontánea. Mientras programar significa comprender la lógica del lenguaje de programación que utilizan, anticipar los comportamientos que desean observar en sus prototipos y lograr utilizar las tareas y comandos apropiados para lograrlos.

En este eje las medias más bajas se observan en la Programación de estructuras de control de tiempo, en donde la principal dificultad se presenta cuando los estudiantes deben utilizar fracciones de tiempo (segundos) para efectos que ocurren muy rápido, la programación de multitareas, pues muchas veces programan secuencias de acciones y no tareas simultáneas que podrían hacer más naturales los comportamientos de los prototipos y la efectividad de los programas que refiere a lograr a cabalidad los efectos programados.

Se realizó una prueba t entre las medias de programación de las ejecuciones 2 y 3 de la propuesta, los resultados muestran que existen diferencias significativas en las medias de la Programación de estructuras de control de tiempo, $\mathrm{t}_{(13)}=-1.55, p=.001$ y el uso de diversidad de comandos $\mathrm{t}_{(12)}=-2.26, p=.001$, evidenciando mejores resultados en la tercera aplicación.

\subsubsection{Proyecto}

En la primera ejecución de la propuesta únicamente se valoró el diseño previo de los prototipos elaborados por los estudiantes y fue uno de los aspectos más bajos en la evaluación, pues por una parte los y las estudiantes no están acostumbrados de manera natural a diseñar y planificar sus construcciones y por otra la propuesta no era suficientemente específica sobre este aspecto, de manera que los docentes tampoco propiciaron la realización a cabalidad del proceso de diseño previo. Este es uno de los cambios implementados en la propuesta educativa, en la segunda y en la tercera ejecución. Además, se trabajó este aspecto durante los talleres de capacitación de los docentes de manera que lograran completar el proceso de diseño, previo a iniciar la construcción y programación de los proyectos grupales.

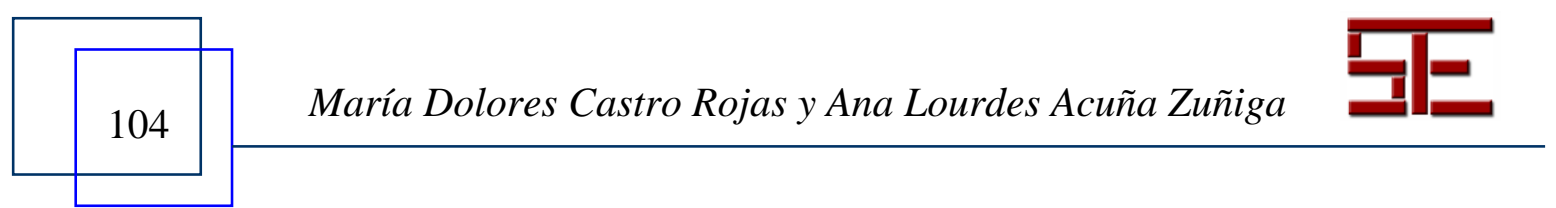




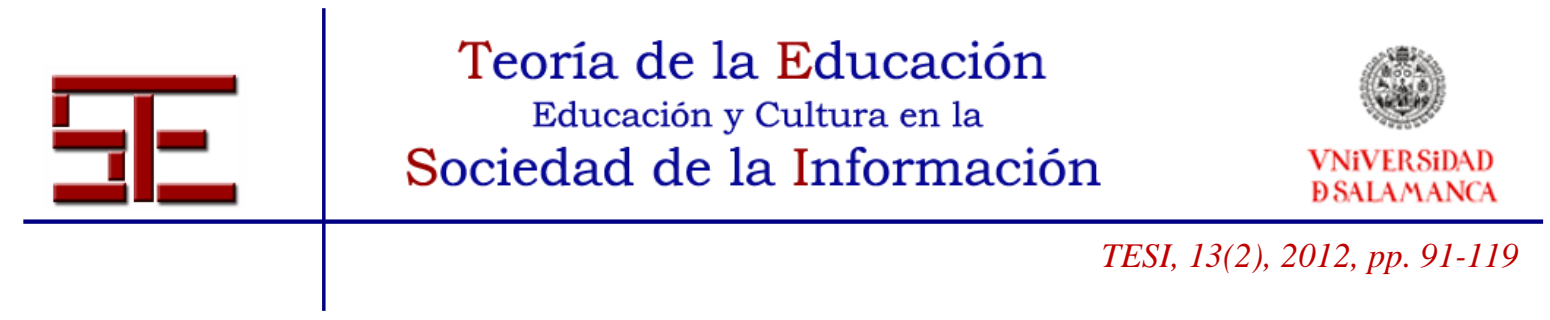

En esta primera exposición los estudiantes se esforzaron por generar cierta unidad temática entre sus construcciones y mostraron diversidad de prototipos, lo que refleja su creatividad e imaginación. Los resultados para este eje se muestran en la Tabla 4.

Tabla 4. Resultados para proyecto grupal en la primera exposición

\begin{tabular}{lcccccc} 
& \multicolumn{3}{c}{$\begin{array}{c}\text { Centros Interactivos } \\
\text { 25 de Julio, } \\
\text { Hatillo }\end{array}$} & $\begin{array}{c}\text { Concepción de } \\
\text { Alajuelita }\end{array}$ & \multicolumn{2}{c}{$\begin{array}{c}\text { Linda Vista } \\
\text { Patarrá }\end{array}$} \\
\cline { 2 - 7 } Aspecto & M & DE & M & DE & M & DE \\
& 4 Sesión & 4 & 5 & 5 & 6 & 6 \\
Diseño & & Sesión & Sesión & Sesión & Sesión & Sesión \\
Temática & 2.75 & .88 & 2.75 & .50 & 3.70 & .94 \\
Diversidad & & & 2.75 & .95 & 4.00 & 0.0 \\
& & & 3.00 & .81 & 4.00 & 0.0
\end{tabular}

En la dimensión "Temática" se evaluó que todos los prototipos guardaran alguna relación con el tema general elegido por el grupo, además que cada robot cumpliera alguna función en el escenario elegido y que conservaran una unidad temática, algo que en términos generales se alcanzó en todas las ejecuciones. La diversidad se refiere a que los prototipos del proyecto general fueran distintos entre sí y mostraran comportamientos diversos, aspecto que también se logró en un nivel aceptable durante las ejecuciones 2 y 3 de la propuesta educativa.

No se pudieron comparar las medias de los tres procesos de aprendizaje, pues en la primera ejecución únicamente se evaluó el diseño previo. Se realizó una prueba t para las medias de la tercera y cuarta sesión y no se encontraron diferencias significativas excepto en el componente de Temática $\mathrm{t}_{(12)}=.41, p=.001$ mostrando una mejoría en la tercera aplicación, en la que se evidenciaron prototipos mejor adaptados y contextualizados a la temática común elegida por el grupo.

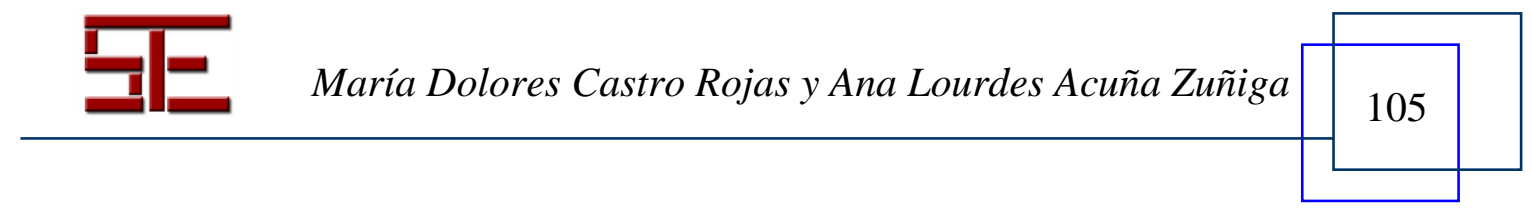




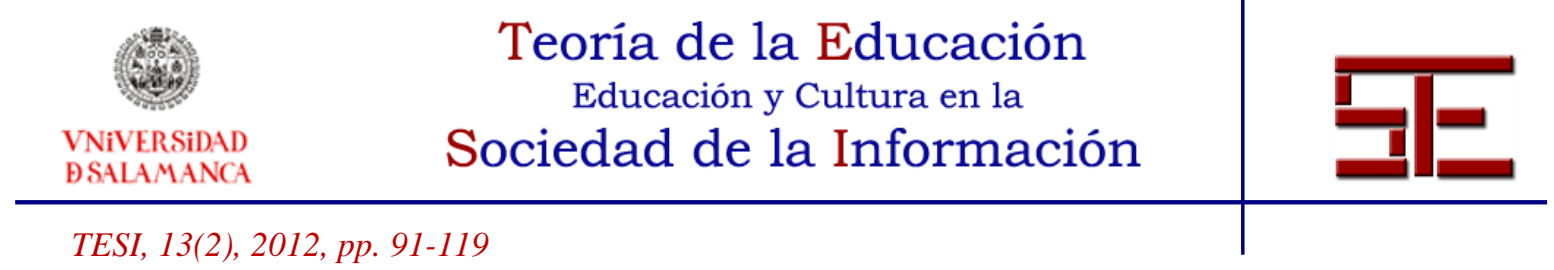

\subsubsection{Exposición}

En las tres ejecuciones se observó que para los y las estudiantes no resulta un proceso natural exponer su trabajo y socializar sus procesos de aprendizaje, cuentan con pocas habilidades para hablar en público, compartir sus ideas y los procesos de pensamiento que realizaron para lograr sus prototipos robóticos. Esto probablemente se debe a que en el entorno en el que se desenvuelven cuentan con pocas oportunidades para poner en práctica estas habilidades y desarrollar esas competencias de comunicación.

Tabla 5. Resultados para la primera exposición de proyectos de las tres aplicaciones

\begin{tabular}{|c|c|c|c|c|c|c|}
\hline \multirow{4}{*}{ Aspecto } & \multicolumn{6}{|c|}{ Centros Interactivos } \\
\hline & \multicolumn{2}{|c|}{$\begin{array}{l}25 \text { de Julio, } \\
\text { Hatillo }\end{array}$} & \multicolumn{2}{|c|}{$\begin{array}{l}\text { Concepción de } \\
\text { Alajuelita }\end{array}$} & \multicolumn{2}{|c|}{$\begin{array}{l}\text { Linda Vista } \\
\text { Patarrá }\end{array}$} \\
\hline & $\mathrm{M}$ & $\mathrm{DE}$ & $\mathrm{M}$ & $\mathrm{DE}$ & $\mathrm{M}$ & $\overline{\mathrm{DE}}$ \\
\hline & $\begin{array}{c}4 \\
\text { Sesión }\end{array}$ & $\begin{array}{c}4 \\
\text { Sesión }\end{array}$ & $\begin{array}{c}5 \\
\text { Sesión }\end{array}$ & $\begin{array}{c}5 \\
\text { Sesión }\end{array}$ & $\begin{array}{c}6 \\
\text { Sesión }\end{array}$ & $\begin{array}{c}6 \\
\text { Sesión }\end{array}$ \\
\hline $\begin{array}{l}\text { Claridad y orden de la } \\
\text { exposición }\end{array}$ & & & 2.75 & .95 & 3.90 & .31 \\
\hline Organización de la información & & & 2.75 & .95 & 3.90 & .31 \\
\hline
\end{tabular}

En la primera ejecución de los talleres se observaron dificultades en los estudiantes para exponer su trabajo, por eso se decidió observar este aspecto y evaluarlo en las siguientes ejecuciones. En la segunda aplicación se observa que las medias obtenidas son bajas en relación con el nivel de desempeño esperado y en relación con las medias obtenidas en los otros ejes evaluados (construcción, programación, proyecto grupal). En la tercera ejecución las medias se acercan al valor máximo esperado 4 mostrando una mejoría respecto a la segunda ejecución. Sin embargo, las diferencias no resultaron estadísticamente significativas al nivel $p=0.001$.

En este punto cabe resaltar que inicialmente la propuesta educativa original no incluyó contenidos en esta dimensión pues se asumió que para los estudiantes ese proceso sería fluido y natural; sin embargo, dentro de las adaptaciones a la propuesta de aprendizaje se incluyó un espacio para que los y las estudiantes planifiquen y practiquen su

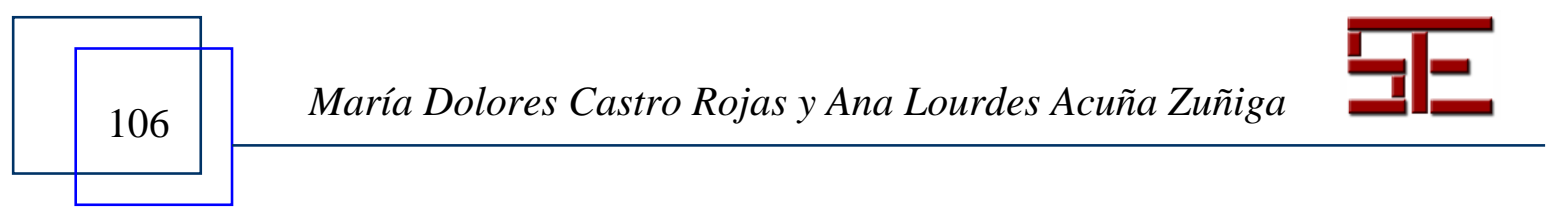




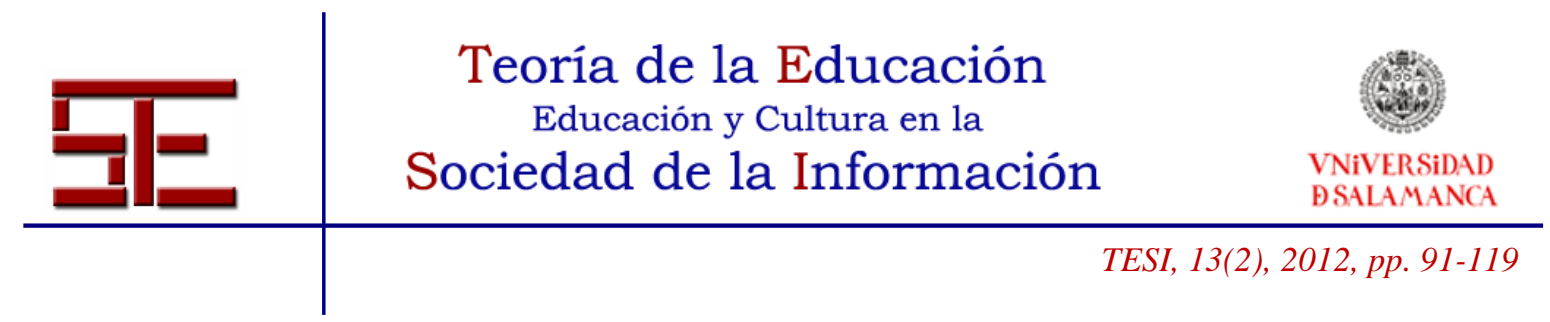

exposición para lo cual se le implementaran estrategias durante los talleres del 2012 y se les brindará material de apoyo.

\subsection{Resultados obtenidos en las exposiciones finales}

Para evaluar los proyectos de los estudiantes al finalizar los talleres también se contó con dos evaluadoras y los resultados se presentan de acuerdo a las dimensiones evaluadas en cada eje de la propuesta educativa. Cabe aclarar que en los primeros talleres (primera ejecución) debido a problemas logísticos no fue posible evaluar las exposiciones finales, por lo que se presentan los resultados únicamente para las ejecuciones 2 y 3 .

\subsubsection{Construcción}

En estas exposiciones los proyectos grupales representaron sitios como lugares donde ocurren actividades particulares y eventos como la representación de sucesos de índole social, que pueden observarse en la realidad, los prototipos robóticos se realizaron en subgrupos de estudiantes y guardaron unidad temática elegida por el grupo general, por ejemplo se observaron: festivales, fiestas populares, eventos deportivos, situaciones de rescate y celebraciones.

En la Tabla 6 se observa que las medias de construcción superan el valor 3 que representa un nivel aceptable de desempeño en los estudiantes.

Tabla 6. Resultados en construcción exposición final para la segunda y tercera ejecución de la propuesta

\begin{tabular}{|c|c|c|c|c|}
\hline \multirow[t]{4}{*}{ Aspecto } & \multicolumn{2}{|c|}{ Alajuelita } & \multicolumn{2}{|c|}{$\begin{array}{l}\text { Linda Vista } \\
\text { Patarrá }\end{array}$} \\
\hline & $\mathrm{M}$ & $\overline{\mathrm{DE}}$ & $\mathrm{M}$ & DE \\
\hline & 10 & 10 & 12 & 12 Sesión \\
\hline & Sesión & Sesión & Sesión & \\
\hline $\begin{array}{l}\text { Conocimiento y utilización de } \\
\text { piezas de construcción }\end{array}$ & 3.56 & .62 & 3.75 & .62 \\
\hline Utilización de mecanismos & 3.65 & .49 & 3.75 & .42 \\
\hline $\begin{array}{l}\text { Utilización de sistemas de } \\
\text { engranajes }\end{array}$ & 3.13 & .61 & 3.42 & .66 \\
\hline $\begin{array}{l}\text { Resolución de problemas de } \\
\text { construcción }\end{array}$ & 3.53 & .62 & 3.67 & .49 \\
\hline Uso del tiempo & 3.53 & .64 & 3.58 & .79 \\
\hline
\end{tabular}

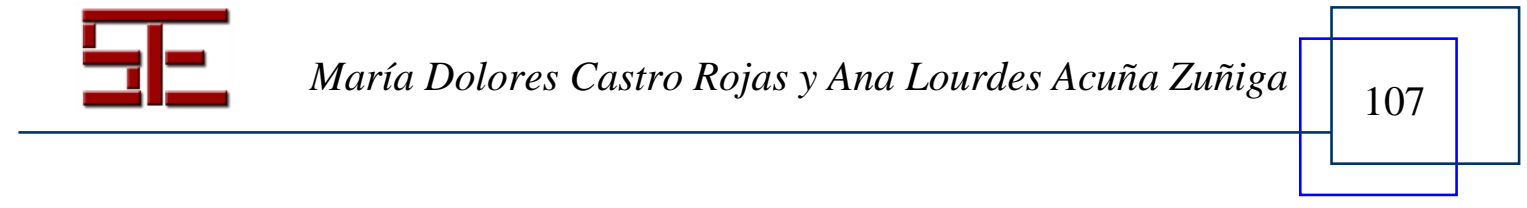




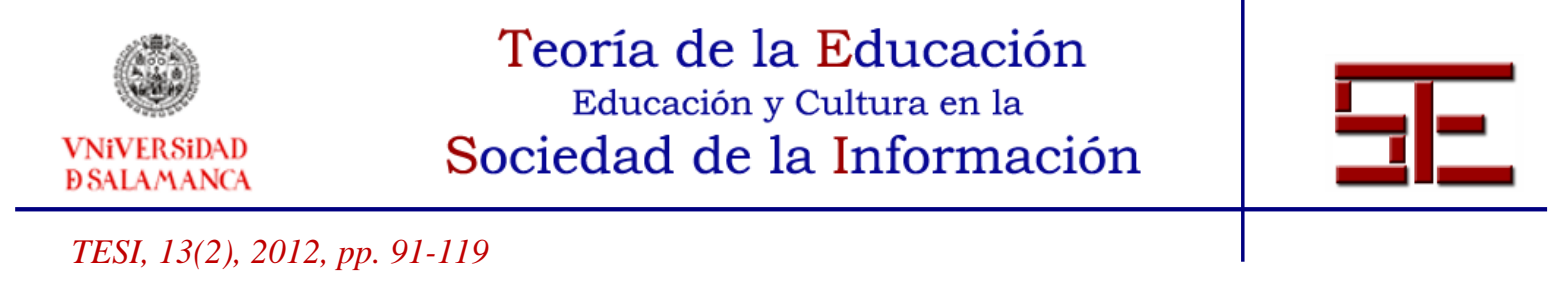

Cabe destacar que en los proyectos finales se observó la aplicación de conceptos complejos de construcción como grados de libertad y la utilización de mecanismos complejos integrados por diferentes máquinas y operadores para la transmisión de movimiento (sistemas de engranajes y poleas compuestos, levas, tornillos, excéntricas).

Sin embargo, se observa que en ambas ejecuciones el aspecto con menor puntaje es la utilización de sistemas de engranajes esto se observó sobre todo cuando las construcciones requieren sistemas de engranajes compuestos, los estudiantes tienen problema con este aspecto pues no logran anticipar en sus diseños los requerimientos mecánicos de sus prototipos para que cumplan con las funciones que les asignan. En muchos casos tratan de resolver todos los requerimientos utilizando sistemas de engranajes simples, sin embargo, ese tipo de mecanismos no responden a las funcionalidades que desean. Se compararon las medias de ambas sesiones y no se encontraron diferencias estadísticamente significativas.

\subsubsection{Programación}

En el ámbito de la programación también se evidenciaron mejoras conforme se fue consolidando la propuesta educativa y el equipo docente adquirió más experiencia con el trabajo y los contenidos. Para las exposiciones finales la programación de los proyectos integró de manera casi generalizada estructuras de programación con multitareas, ciclos condicionados, mayor control del tiempo y la optimización del uso de sensores. Asimismo, los estudiantes integraron mayor cantidad y diversidad de sensores incluyendo sensores que valoran el tacto, temperatura y luz. En la Tabla 7 se muestran los resultados para este eje.

Tabla 7. Resultados en programación exposición final para la segunda y tercera ejecución de la propuesta

\begin{tabular}{ccccc}
\hline \multirow{2}{*}{$\begin{array}{c}\text { Aspecto } \\
\end{array}$} & \multicolumn{2}{c}{$\begin{array}{c}\text { Concepción de } \\
\text { Alajuelita }\end{array}$} & \multicolumn{2}{c}{ Linda Vista Patarrá } \\
\cline { 2 - 5 } & $\mathrm{M}$ & $\mathrm{DE}$ & $\mathrm{M}$ & $\mathrm{DE}$ \\
& 10 & 10 & 12 Sesión & 12 Sesión \\
& Sesión & Sesión & & \\
\cline { 2 - 5 } & 3.76 & .43 & 4.00 & .00 \\
Programación de actuadores & 3.47 & .80 & 3.67 & .49 \\
$\begin{array}{c}\text { Resolución de problemas de } \\
\text { programación }\end{array}$ & & & & \\
\hline
\end{tabular}

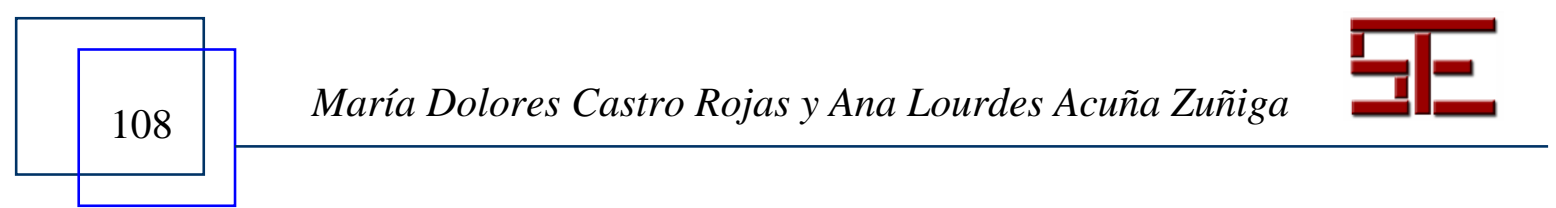




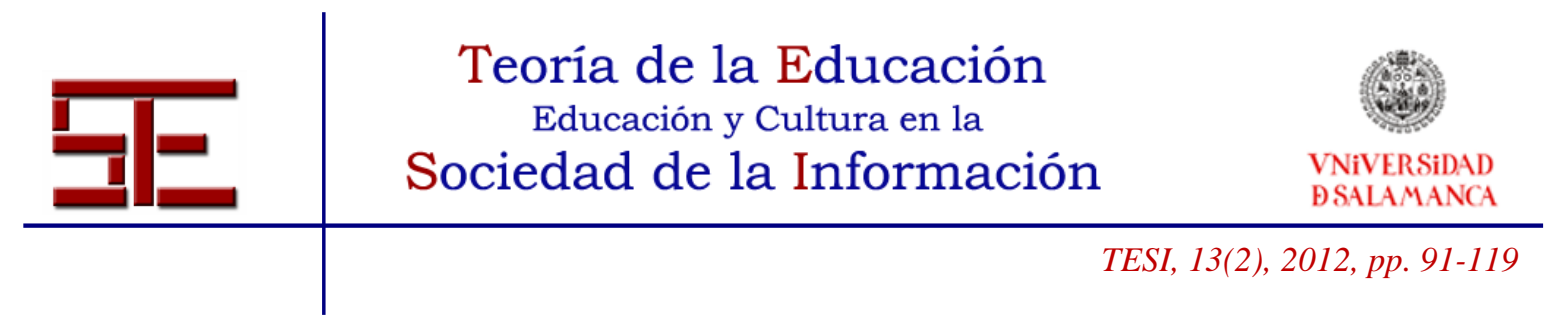

\begin{tabular}{ccccc}
\hline $\begin{array}{c}\text { Programación de estructuras de } \\
\text { control de tiempo }\end{array}$ & 3.71 & .58 & 3.75 & .62 \\
$\begin{array}{c}\text { Uso de estructuras de } \\
\text { programación }\end{array}$ & 2.29 & .68 & 3.33 & .77 \\
$\quad$ Utilización de tareas & 3.00 & 1.1 & 3.83 & .57 \\
Diversidad de comandos & 3.41 & .870 & 3.92 & .28 \\
$\quad$ Efectividad & 3.65 & .606 & 3.67 & .49 \\
\hline
\end{tabular}

Se observa que de manera sistemática muchos de los valores de las medias de programación fueron más bajos que las medias en construcción. En este sentido, cabe mencionar que si bien es cierto que los chicos y chicas visualizan claramente en la construcción el componente lúdico y se les facilita la anticipación de las piezas que podrían utilizar para resolver los problemas que van enfrentando. En la programación estas estrategias no resultan tan efectivas y es requerido transcender los procesos intuitivos por procesos más analíticos y de conocimiento que, aunque fueron estudiados durante el desarrollo de la propuesta, no siempre alcanzaron niveles de comprensión lo suficientemente fluidos para trascender su uso a otros contextos o situaciones. Algunas modificaciones a la propuesta para el año siguiente incluyen más espacios de práctica y resolución de retos de programación.

En términos generales, los valores para este aspecto se mantuvieron por encima del valor 3, lo que indica un buen desempeño de los estudiantes, logrando crear programas para que sus prototipos cumplan con las conductas y las funciones propuestas y de acuerdo a las necesidades que los diseños construidos demandaban.

De manera específica en la segunda y la tercera ejecución la media más baja fue el uso de estructuras de programación que se refiere a la incorporación de estructuras iterales y condicionales utilizando diversos sensores y comandos. Al explorar sobre las razones por las cuales los estudiantes se les dificulta alcanzar el nivel esperado se concluyó que se debió a las limitaciones de tiempo para realizar más prácticas y resolver problemas que les permitan ganar mayor comprensión y fluidez en el uso de estos conceptos. Ante esta situación se decidió excluir el tema de estructuras condicionales e incluirlo en una segunda propuesta educativa que será diseñada en el año 2012, y mantener únicamente el de las estructuras itérales para este primer nivel de robótica.

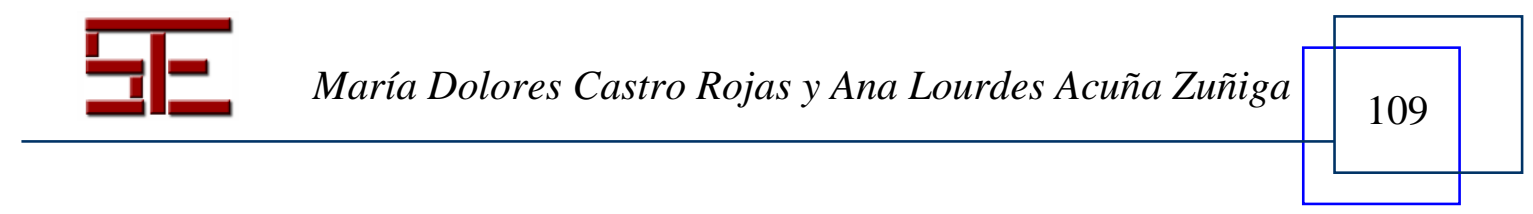




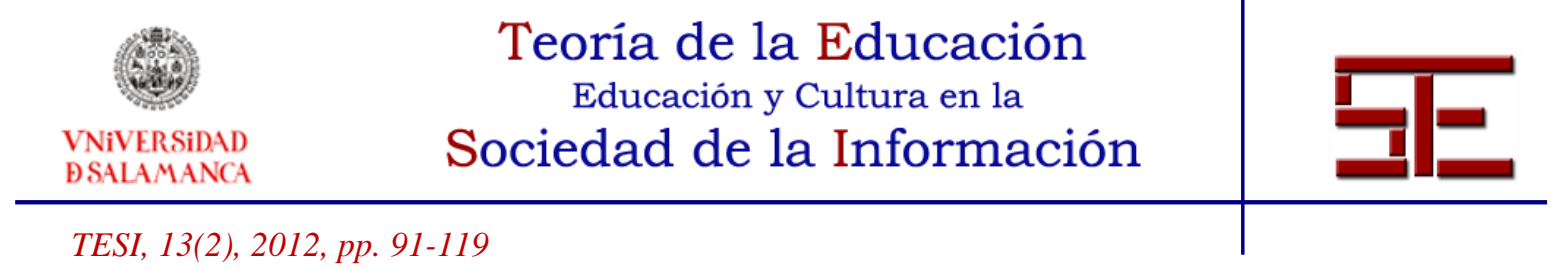

Finalmente al comparar las medias de las dos ejecuciones, únicamente se encontraron diferencias significativas en la Programación de actuadores $\mathrm{t}_{(26)}=-1.77, p<.001$, donde se observa un mejor desempeño en la tercera ejecución y en el uso de Diversidad de comandos $\mathrm{t}_{(27)}=-1.77, p=.002$, mostrando que en la tercera ejecución del taller los estudiantes utilizaron mayor variedad de recursos en sus proyectos.

\subsubsection{Proyecto grupal}

Los proyectos finales requerían ser proyectos planeados y diseñados en forma grupal, de manera que las personas participantes eligen una situación problema por resolver o una temática por representar. A partir de esa elección la resolución de la situación o la representación se divide en partes y se conforman grupos de participantes que se responsabilizan de la creación de cada una de las partes. Todas las representaciones deben incluir los conceptos estudiados a nivel de construcción, programación y muestras de interacción e inteligencia a través de la captura y manipulación de la información por medio de sensores. Cada integrante de los subgrupos tiene tareas que por consenso se asignan luego de concretar el diseño y los requisitos.

En la segunda ejecución los estudiantes de uno de los grupos realizaron una representación de los juegos olímpicos que incluyó la recreación de diversas disciplinas deportivas (tenis, natación, otros) y otro grupo representó un zoológico que incluyó prototipos de animales (caballo, jirafa, otros), en esta ocasión todos los prototipos fueron robots caminantes y con varios grados de libertad en sus movimientos que conllevan mayor complejidad en la construcción, el acople y la estabilidad de sus estructuras.

En la tercera ejecución de la propuesta se observó la representación de los festejos populares de fin de año con prototipos de juegos mecánicos (ruedas de Chicago, torre y tornados) y la simulación de una operación de rescate de un grupo de sobrevivientes atrapados en una montaña, allí se incluyeron helicópteros, teleférico, maquinaria especializada para remover escombros y abrir camino (tractores y grúas).

En ambas exposiciones se observaron trabajos de buena calidad que respondían de manera coherente a una temática definida por el grupo y que mostraban coherencia y secuencialidad entre las acciones representadas. Los prototipos de cada uno de los subgrupos o parejas cumplían con una función en el eje temático elegido por el grupo y los robots mostraron identidad propia en su forma física y en la programación de sus comportamientos.

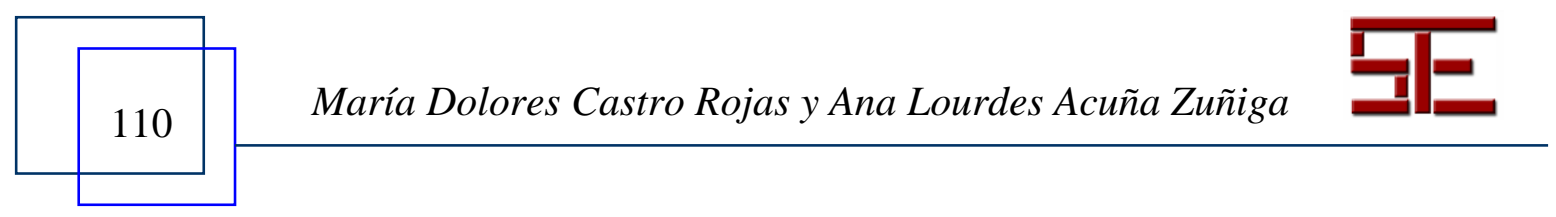




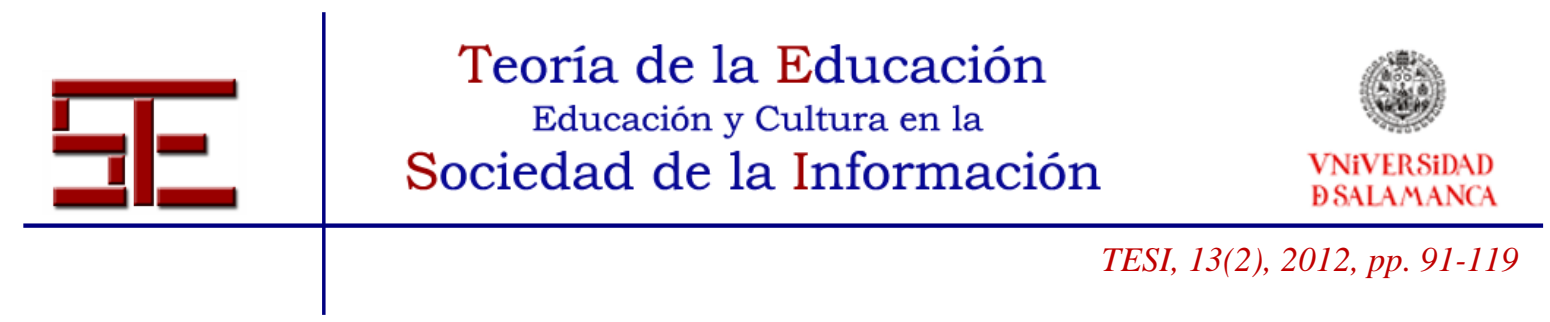

Todas las medias en este aspecto se ubicaron en un valor cercano a 4, lo que refleja que los estudiantes en la mayoría de los casos alcanzaron el desempeño esperado para los proyectos finales. Los resultados se observan en la Tabla 8.

Tabla 8. Resultados en el proyecto final para la segunda y tercera ejecución de la propuesta

\begin{tabular}{ccccc}
\hline Aspecto & \multicolumn{2}{c}{$\begin{array}{c}\text { Concepción de } \\
\text { Alajuelita }\end{array}$} & \multicolumn{2}{c}{$\begin{array}{c}\text { Linda Vista } \\
\text { Patarrá }\end{array}$} \\
\cline { 2 - 5 } & $\mathrm{M}$ & $\mathrm{DE}$ & $\mathrm{M}$ & $\mathrm{DE}$ \\
& 10 & 10 & 12 Sesión & 12 \\
& Sesión & Sesión & & Sesión \\
\cline { 2 - 5 } & 3.75 & .45 & 4.00 & .00 \\
Diseño & 4.00 & .00 & 4.00 & .00 \\
Temática & 4.00 & .00 & 4.00 & .00 \\
Diversidad & & & & \\
& & & & \\
\hline
\end{tabular}

Se observa que en la tercera ejecución mejoró de manera significativa el ámbito de diseño $t_{(17)}=-1.44, p<.001$, pues por la complejidad de los proyectos fue necesario que los estudiantes diseñaran previamente su proyecto y los componentes que lo integran, primero de manera conjunta el proyecto general y luego los prototipos de cada subgrupo. Estos resultados evidencian un abordaje más sistemático de este aspecto en el proceso de los talleres y la fase previa de diseño se plasma en la calidad y la diversidad de las producciones observadas.

\section{Exposición}

Pese a que los proyectos de la exposición final, fueron productos de alta calidad y complejidad, los estudiantes demostraron pocas habilidades para hablar en público, verbalizar sus procesos de pensamiento y las secuencias del trabajo que realizaron. En el momento de exponer presentaron la información de manera desorganizada y fue evidente que los estudiantes no prepararon la exposición. Esta situación podría ser consecuencia de la falta de planificaciòn por parte del docente para que los estudiantes

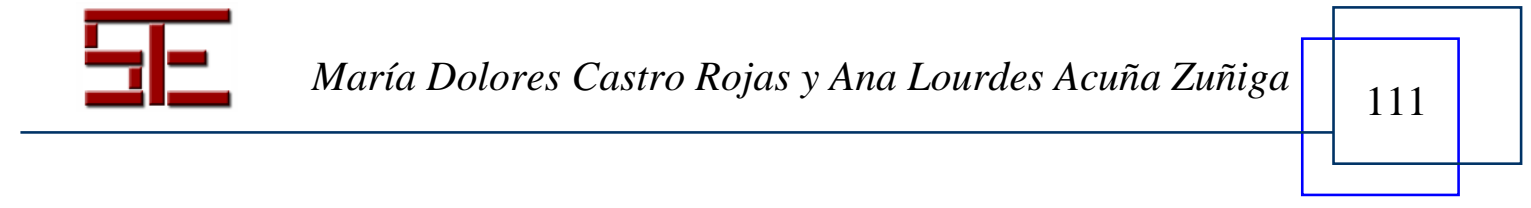




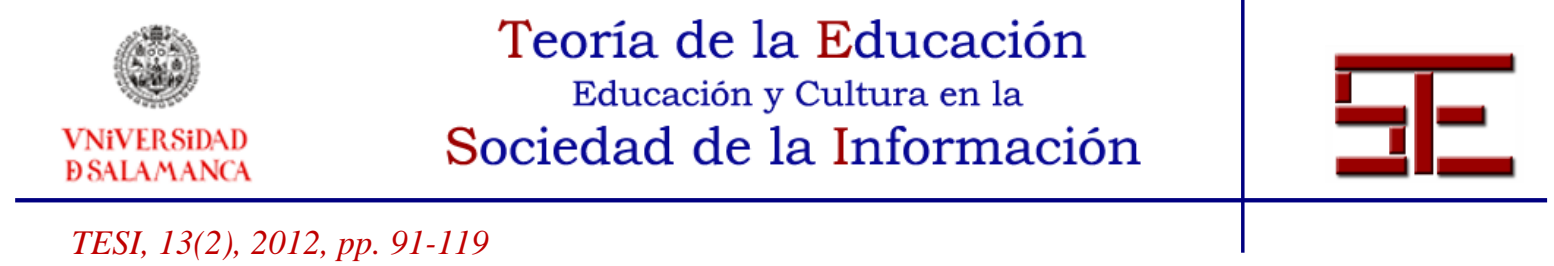

planeen su exposición de acuerdo a criterios establecidos. También puede haber afectado el tiempo que los estudiantes que dedican al perfeccionamiento de sus diseños robóticos para que realicen con efectividad sus funciones y comportamientos, relegando espacio y tiempo para planificar la exposición. Cabe destacar que como parte del proceso exposición y divulgación de los proyectos se incluye el registro de la experiencia en blogs que los estudiantes van haciendo de forma paralela con su proyecto final. Todos los grupos alcanzaron esta meta. En las primeras exposiciones las docentes intervinieron significativamente en los procesos de edición delegando conforme fueron adquiriendo mayor dominio de esta aplicación en estudiantes que asumieron las tareas de registro, recuperación y publicación de sus producciones.

Aunque se observan medias bajas para los aspectos relacionados con la exposición asociados a la claridad, orden y organización de ideas (ver tabla 9) para el segundo año de ejecución de esta propuesta educativa se trabajará para mejorar las habilidades de los y las estudiantes en este ámbito.

Tabla 9 Resultados para la exposición final para la segunda y tercera ejecución de la propuesta

\begin{tabular}{ccccr}
\hline & \multicolumn{2}{c}{$\begin{array}{c}\text { Concepción de } \\
\text { Alajuelita }\end{array}$} & \multicolumn{2}{c}{ Linda Vista Patarrá } \\
\cline { 2 - 5 } Aspecto & $\begin{array}{c}\text { M } \\
\text { 10 Sesión }\end{array}$ & $\begin{array}{c}\text { DE } \\
\text { 10 Sesión }\end{array}$ & $\begin{array}{c}12 \\
\text { Sesión }\end{array}$ & $\begin{array}{c}12 \\
\text { Sesión }\end{array}$ \\
\hline $\begin{array}{c}\text { Claridad y orden de la } \\
\text { exposición }\end{array}$ & 3.76 & .437 & 2.88 & .83 \\
$\begin{array}{c}\text { Organización de la información } \\
\text { Organy }\end{array}$ & 3.69 & .479 & 2.75 & .70 \\
\hline
\end{tabular}

Aunque se observan diferencias entre el nivel de desempeño alcanzado por los estudiantes en la segunda y tercera aplicación, esas diferencias no resultaron estadísticamente significativas.

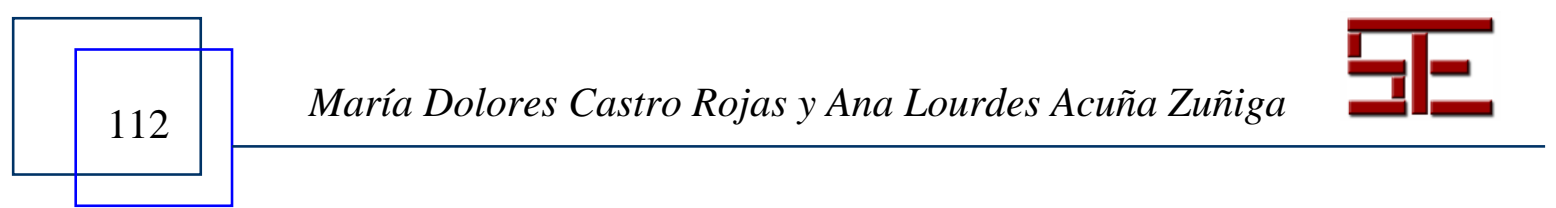




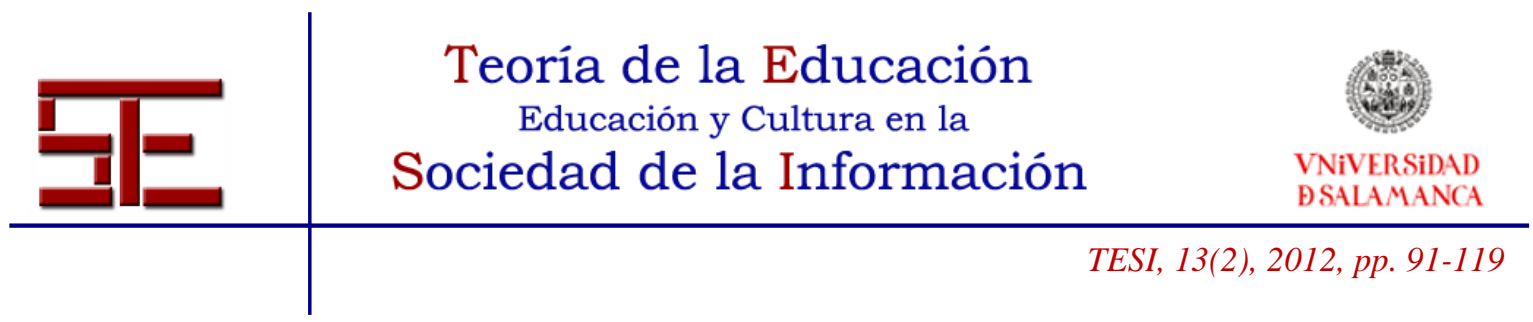

\section{5.- SÍNTESIS DE RESULTADOS}

Respecto a la propuesta educativa que fue diseñada para este proyecto, se observa que al primer año de ejecución las educadoras reconocen los énfasis de comprensión que se espera que los estudiantes logren y han orientado su práctica educativa hacia ese fin. La propuesta de aprendizaje evidencia un orden lógico de ejecución que hace posible su implementación en el tiempo estimado, independientemente de los grupos con que se ejecute, siempre que se cuente con una cantidad de recursos tecnológicos y espacio físico adecuados. Será necesario mejorar los materiales de aprendizaje que se muestran a los estudiantes y la inclusión de prácticas de algunos conceptos de construcción y programación. También será necesario que los docentes continúen sus procesos de capacitación en este campo.

Los aspectos que se deben reforzar serán retomados y analizados antes de implementar los talleres de robótica educativa en FundaVida en el 2012, con el fin de implementar las estrategias pedagógicas y los materiales educativos pertinentes para alcanzar los desempeños esperados en los estudiantes.

Durante las tres ejecuciones de los talleres a lo largo del año 2011, de manera sistemática se observaron mejoras en el desempeño de los estudiantes en el área de construcción. El conocimiento y familiaridad con las piezas, los principios mecánicos y la resolución de problemas posibilitaron que los prototipos de las exposiciones finales fueran más elaborados y se acercaran notablemente a las características esperadas al finalizar la participación en la propuesta educativa.

En términos generales los resultados del proceso de evaluación muestran lo siguiente:

Al finalizar la primera mitad del taller:

- Los estudiantes pueden construir prototipos simples, utilizando piezas de construcción genéricas.

- Utilizan mecanismos simples para generar movimiento.

- Aplican conceptos de fuerza y velocidad en sus prototipos.

- Programan estructuras simples e iterales para el control de actuadores y del tiempo.

- Trabajan en parejas para hacer sus proyectos.

- Incorporan sensores de tacto y luz en diferentes apartados de los programas.

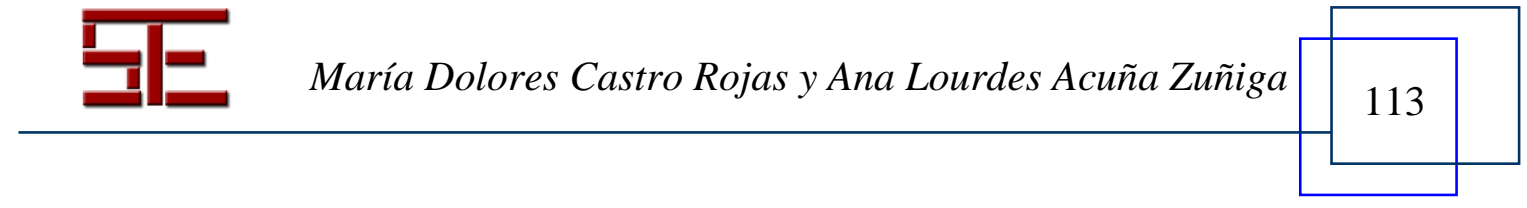




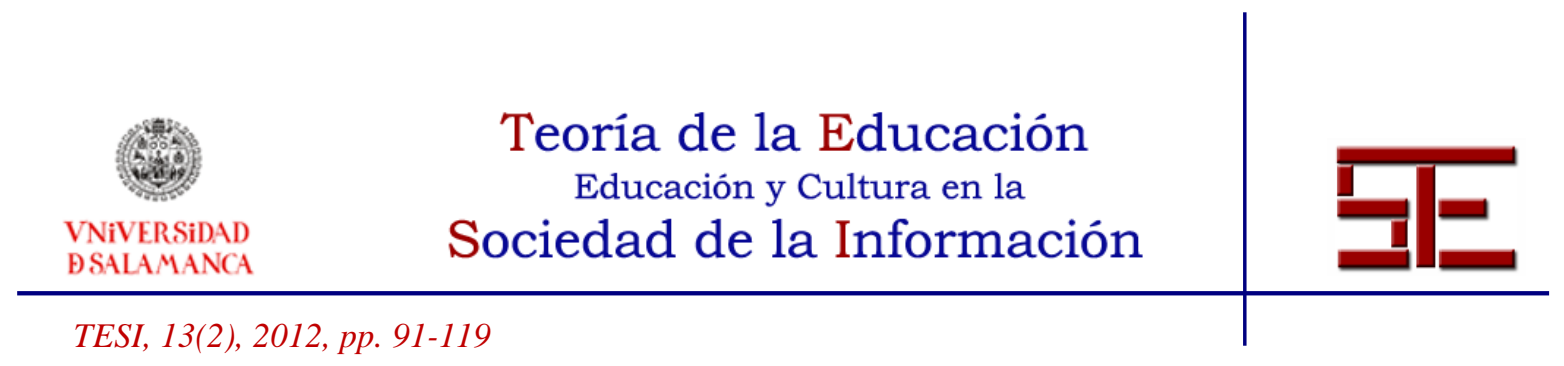

Se deben reforzar las siguientes áreas:

- El diseño previo de los prototipos.

- Construcción de prototipos más estables y robustos.

- Comprensión de principios mecánicos y utilización de operadores que generen transmisión de movimiento produciendo intencionalmente fuerza o velocidad.

- Mayor comprensión de la lógica de las estructuras de control de actuadores, tiempo y tareas.

- Utilización de estructuras de programación complejas (ciclos condicionados, multitareas).

Al momento de finalizar la propuesta educativa los estudiantes pueden:

- Identifica los principios constructivos y mecánicos que les permiten crear y poner a funcionar prototipos robóticos de invención propia.

- Elaborar proyectos coordinados de manera grupal para representar sitios o eventos que pueden observarse en la realidad. Las producciones guardan una unidad temática, y cada robot tiene identidad propia en su diseño y funcionamiento, por ejemplo: parques de diversiones, festivales, situaciones de rescate, celebraciones.

- Aplicar conceptos complejos en el eje de construcción tales como grados de libertad. Además utilizan mecanismos complejos integrados por diferentes máquinas y operadores para la transmisión de movimiento (sistemas de engranajes y poleas compuestos, levas, tornillos, excéntricas).

- Integrar en sus proyectos mayor cantidad y diversidad de sensores incluyendo algunos que valoran el tacto, temperatura y luz en tareas que se ejecutan de forma paralela.

- Incluir ciclos condicionados y multiprocesos en sus programas.

- Desarrollar la programación de manera más elaborada, incluyendo casi en todos los programas estructuras para multitareas y ciclos condicionados.Además, logra un mayor dominio de las estructuras de control del tiempo y optimizan del uso de sensores.

- Compartir sus producciones en forma verbal y publicar sus proyectos en formatos digitales.

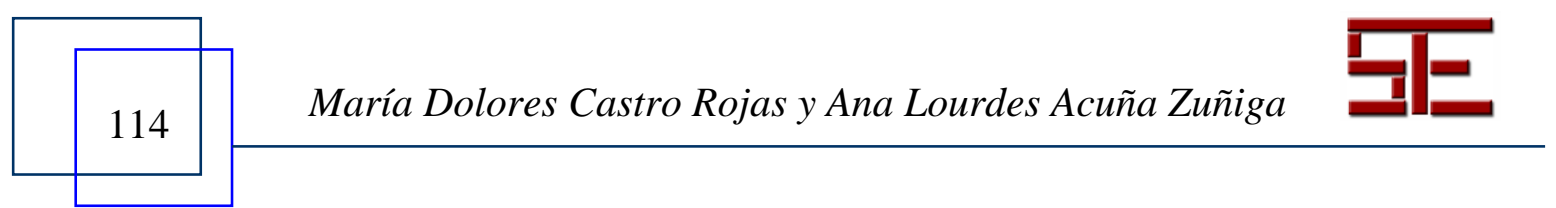




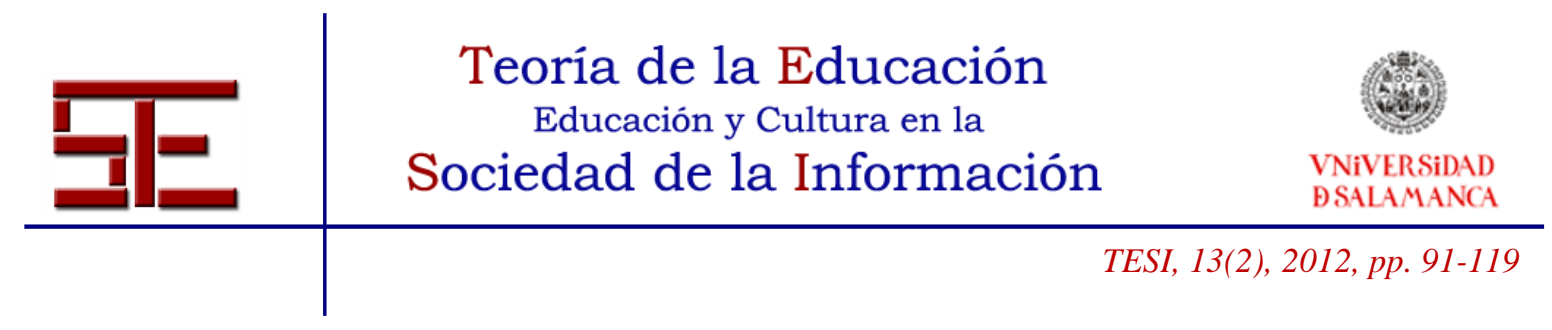

Se deben reforzar:

- Las estrategias de resolución de problemas tanto a nivel tecnológico como de convivencia y aprendizaje con sus pares.

- La fase de diseño previo del proyecto y la distribución de tareas entre los miembros integrantes de cada subgrupo.

- La comprensión y anticipación del uso de mecanismos con sistemas de engranes compuestos para prototipos complejos.

- El uso de estructuras condicionales en la programación.

- Mayor integración entre los elementos de los proyectos grupales.

- El desarrollo de habilidades para hablar en público y expresar sus ideas de manera clara y ordenada aprovechando los recursos digitales creados para ese fin.

\section{6.- CONCLUSIONES}

Los resultados obtenidos permiten afirmar que, mediante la participación en un proceso de aprendizaje con robótica educativa, los estudiantes de FundaVida comprenden los principios fundamentales de la robótica y del diseño de proyectos e identifican y ponen en práctica estrategias de construcción, programación y control de prototipos robóticos de invención propia. Asimismo, la propuesta educativa diseñada e implementada en los talleres, acerca a los participantes al mundo de la tecnología y les brinda la posibilidad de ampliar sus horizontes desde aspectos meramente técnicos como son el diseño, construcción y programación de prototipos robóticos, como en las áreas sociales y de divulgación de resultados y productos como por ejemplo: el trabajo en equipo, la comunicación y publicación. Todas estas acciones buscan superar la brecha digital y generar intereses e inquietudes que en el largo plazo pueden materializarse en proyectos de desarrollo profesional.

El participar en procesos de aprendizaje enriquecidos a través del uso de la robótica y de otras tecnologías digitales logra motivar a los estudiantes y generar en ellos compromiso con sus propios procesos de aprendizaje y con el cumplimiento de la oferta educativa. Pues a pesar de que los talleres se ofrecen fuera del horario escolar de los participantes, la mayoría de ellos asistió a la totalidad de las sesiones de manera puntual y realizó las actividades con interés y entusiasmo. Asimismo, los participantes afirman que estarían interesados en participar en nuevas experiencia con robótica educativa. Este

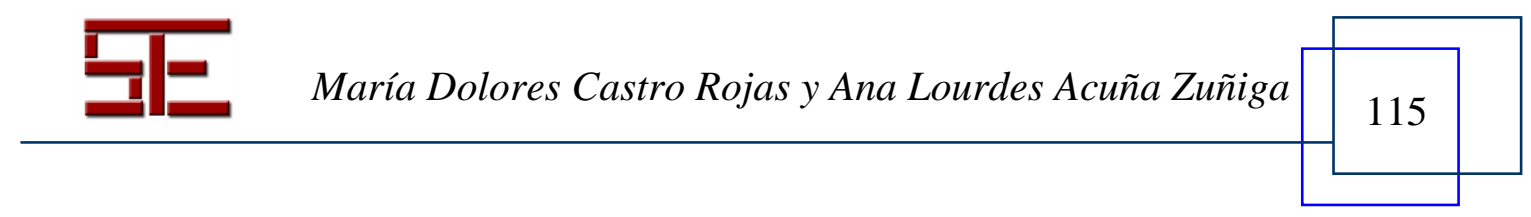




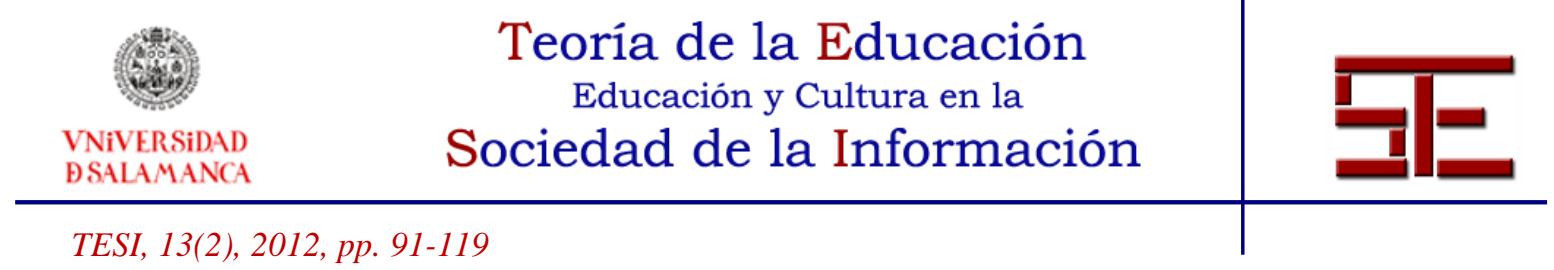

es un logro del proyecto, pues los estudiantes se desenvuelven en sistemas comunales y familiares en los que no se visualiza la educación como motor de cambio de las condiciones socioeconómicas.

En este sentido, también se valora el hecho de que el tiempo que los niños, niñas y adolescentes asisten a los Centros Interactivos es tiempo en el que se encuentran en ambientes seguros y protegidos de los riesgos que se encuentran en las calles de sus barrios y comunidades caracterizados por situaciones de inseguridad, drogas y violencia.

En cuanto a las habilidades creativas y de diseño, se encontró que los participantes conciben y simulan sitios o eventos que pueden ser observados en la realidad, sin embargo, no les resulta natural el proceso de planificación y diseño previo de sus proyectos; en muchas ocasiones los estudiantes se comprometen directamente en el proceso de construcción de sus prototipos, implementando ensayos de prueba y error, lo que les dificulta concretar sus producciones y anticipar los problemas que podrían enfrentar en el proceso. En otras oportunidades realizan el proceso de planificación y diseño previo de manera muy general, lo que no les permite anticipar posibles problemas y formas de solucionarlos. Las habilidades de planificación y diseño previo fueron una de las áreas en las que los estudiantes alcanzaron un menor nivel de logro y que se deben reforzar en una próxima fase del proyecto.

A través de la participación en las sesiones de los talleres, los estudiantes se acercan a conceptos complejos de construcción y programación que logran comprender para luego integrarlos en sus construcciones robóticas evidenciando progresivamente un uso óptimo de las piezas y los operadores mecánicos integrados en máquinas que actuaban de acuerdo a su estructura y funcionamiento.

En la construcción de prototipos los estudiantes alcanzaron un nivel de desempeño satisfactorio, cuando se trata de estructuras simples identifican los principios constructivos y mecánicos que les permiten crear y poner a funcionar prototipos robóticos. Sin embargo, se observan dificultades cuando se trata de estructuras más complejas que requieren el uso de sistemas de engranajes compuestos y grados de libertad. Se deben reforzar los contenidos relacionados con conceptos como fuerza y velocidad que subyacen a los principios de construcción de mecanismos.

Los estudiantes también alcanzaron un nivel de desempeño satisfactorio en las habilidades de programación, dotando a sus robots de sistemas de control que les permiten un buen funcionamiento de acuerdo a su estructura y su función. Sus

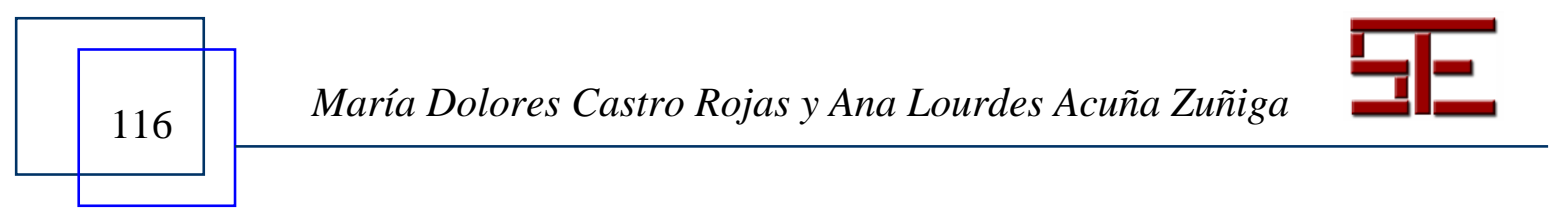




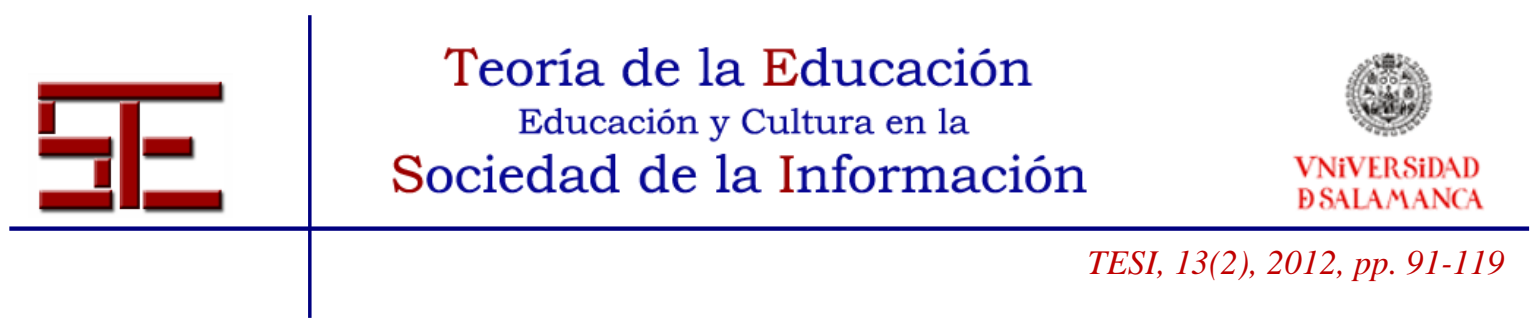

programas incluyen estructuras con multitareas y ciclos condicionados, estructuras de control de tiempo y el uso de sensores.

La resolución de problemas de construcción y programación fue una de las dimensiones en las que hubo menor nivel de logro, pues los estudiantes no consiguen anticipar los problemas que podrían enfrentar en sus proyectos y una vez que están ante situaciones problemas, si bien es cierto que logran generar alternativas de solución, en muchas oportunidades no alcanzan por sí mismos la solución más acertada. Este es uno de los aspectos que se deben mejorar en la propuesta pedagógica, pues originalmente no se incluyó un enfoque de solución de problemas. Sin embargo, con base en las observaciones y evaluaciones realizadas resulta importante fortalecer las fases de diseño previo, e incluir una estrategia específica para la resolución de problemas dentro de la propuesta.

Paradójicamente se observó un bajo nivel de logro de las habilidades sociales. En cuanto al trabajo en equipo los chicos y chicas muestran algunas limitaciones para relacionarse cuando deben trabajar con personas que no conocen, en esos casos el trabajo resulta difícil pues los participantes muestran poca apertura para conocer e interactuar con nuevas personas. Además, se evidencian algunas limitaciones de comunicación que les permitan negociar posiciones y llegar a acuerdos. Por otra parte, en los procesos de divulgación de los procesos de aprendizaje y proyectos finales se observó que los participantes cuentan con pocas habilidades para hablar en público y expresar sus ideas de manera clara y ordenada. En este sentido, es posible pensar que los niños, niñas y adolescentes participantes no cuenten en el contexto en el que se desarrollan con suficientes oportunidades para desarrollar y practicar esas habilidades, por lo que el participar en los talleres de robótica educativa, puede representar una oportunidad importante al fomentar esas destrezas y brindar espacios para su práctica por parte de los estudiantes. En el mismo sentido será necesario dedicar más tiempo para que ordenen las ideas que desean expresar y preparen $\mathrm{y}$ ensayen sus presentaciones.

Cabe destacar que los resultados evidencian que si bien es cierto que se trata de un proyecto para desarrollar habilidades y fluidez tecnológica en los estudiantes, es necesario lograr un balance con el desarrollo de habilidades sociales que les permitan desarrollarse e incorporarse de manera integral en el mundo de la tecnología. Para el desarrollo de las habilidades sociales señaladas se han propuesto distintos recursos y actividades que se implementarán en la segunda fase de ejecución del proyecto durante el 2012.

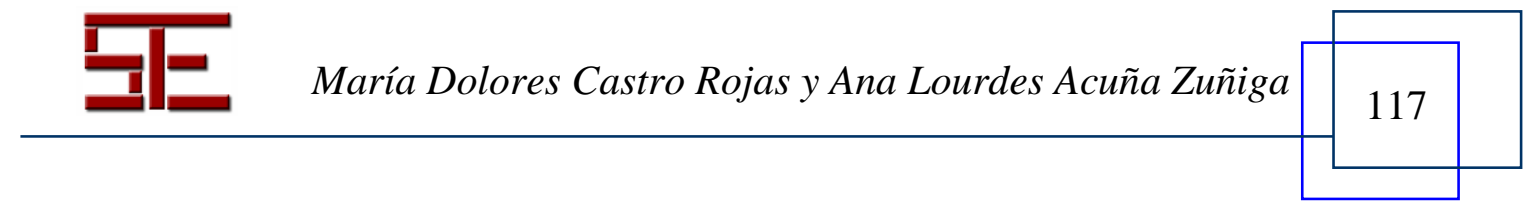




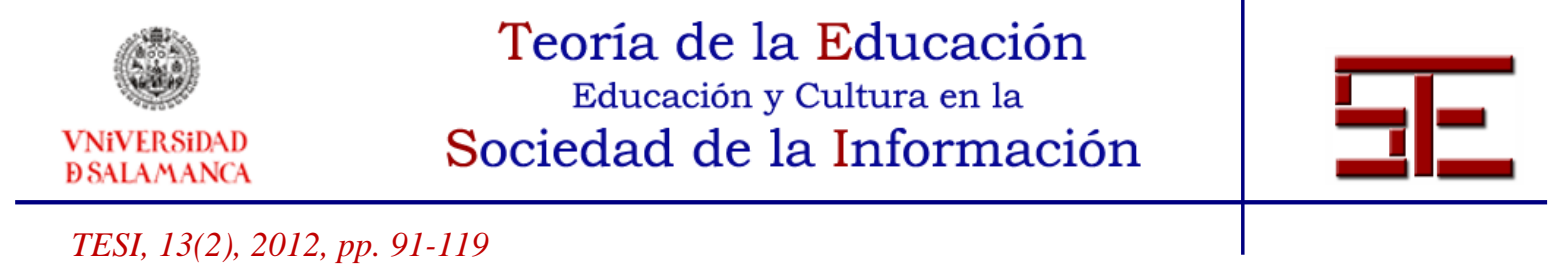

Se debe resaltar que el proceso de evaluación y adaptación realizado durante las ejecuciones de la propuesta educativa permitió incorporar los cambios y ajustes pertinentes a partir de las sesiones de observación y la realimentación por parte de los docentes y estudiantes para lograr una propuesta más adecuada a las características de los y las estudiantes que asisten a los programas de FundaVida. El compromiso mostrado por las docentes y el estudio constante hicieron posible que la implementación de la propuesta se mantuviera con una ejecución estable y coherente con lo propuesto.

Por otra parte, es necesario para una segunda fase de implementación del proyecto incorporar rúbricas de evaluación que se ajusten al momento de desarrollo en el que se encuentra el taller. Si bien es cierto, la rúbrica utilizada durante esta primera fase durante el año 2011 incluyó las habilidades globales que se esperaba observar en los estudiantes, hubo que realizar algunos ajustes pues no es posible observar el mismo desempeño cuando se trata de un nivel intermedio de la propuesta (primera exposición) que en el momento final de los talleres, en el cual se espera que los estudiantes alcancen un mayor nivel de complejidad y profundidad en sus producciones y que las habilidades adquiridas se encuentren más consolidadas.

Finalmente, cabe destacar el esfuerzo del trabajo conjunto de tres organizaciones de distintos sectores sociales que se unieron para brindar oportunidades a una población en riesgo social que de otra forma no contaría con alternativas para insertarse en el mundo de la tecnología y desarrollar las habilidades que se persiguen con este proyecto. Iniciativas de este tipo deben fomentarse en aras de ampliar el universo de oportunidades de esos niños, niñas y adolescentes.

\section{7.- BIBLIOGRAFÍA}

Acuña A. (2006). Robótica: espacios creativos para el desarrollo de habilidades en diseño para niños, niñas y jóvenes en América Latina. Fundación Omar Dengo. Recuperado el 9 de setiembre de 2010, de

http://www.programafrida.net/docs/informes/b66_omardengo.pdf.

Papert, S. (1993). La máquina de los niños. Replantearse la educación en la era de los ordenadores. Barcelona, España: Paidós.

Resnick, M. (2001). Tortugas, termitas y atascos de tráfico. Exploraciones sobre micromundos masivamente paralelos. Barcelona, España: Editorial Gedisa.

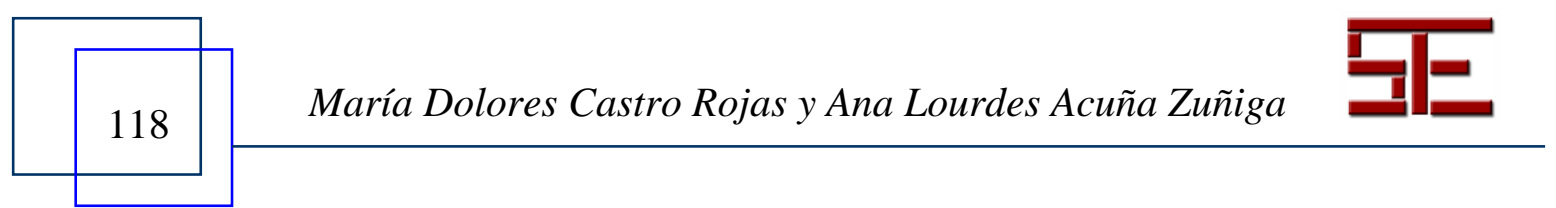




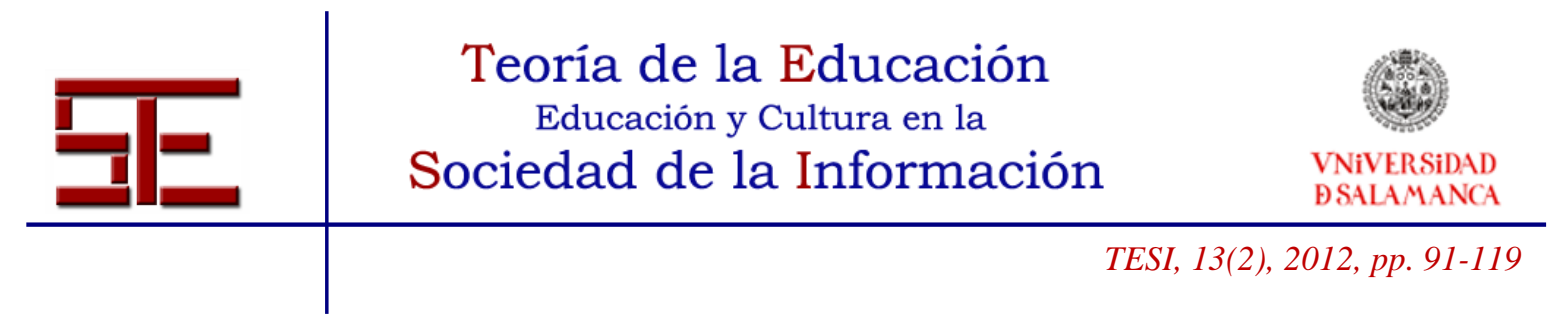

Para citar el presente artículo puede utilizar la siguiente referencia:

Castro Rojas, M. D. y Acuña Zuñiga, A. L. (2012). Propuesta comunitaria con robótica educativa: valoración y resultados de aprendizaje. Revista Teoría de la Educación: Educación y Cultura en la Sociedad de la Información. 13(2), 91-118 [Fecha de consulta: dd/mm/aaaa].

http://campus.usal.es/ revistas_trabajo/index.php/revistatesi/article/view/9001/9246

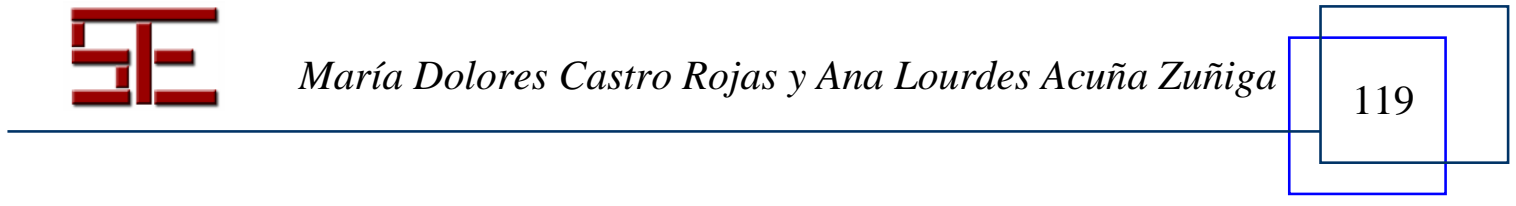

